\title{
Chiral dynamics of kaon-nucleon interactions, revisited
}

\author{
B. Borasoy ${ }^{1 a, b}$, R. Nißler ${ }^{2 a, b}$, W. Weise ${ }^{3 a}$ \\ ${ }^{a}$ Physik Department \\ Technische Universität München \\ D-85747 Garching, Germany \\ ${ }^{b}$ Helmholtz-Institut für Strahlen- und Kernphysik (Theorie) \\ Universität Bonn \\ Nußallee 14-16, D-53115 Bonn, Germany
}

\begin{abstract}
The $\bar{K} N$ system close to threshold is analyzed in view of the new accurate DEAR kaonic hydrogen data. The calculations are performed using chiral SU(3) effective field theory in combination with non-perturbative schemes based on coupled channels. Several variants of such approaches are compared with experimental data and the differences in the results are discussed. Coulomb and isospin breaking effects turn out to be important and are both taken into account. The pole structure of the $\Lambda(1405)$ resonance close to the $\bar{K} N$ threshold is critically examined.
\end{abstract}

PACS: $\quad$ 11.80.-m, 12.39.Fe, 13.75.Jz, 36.10.Gv

Keywords: Chiral Lagrangians, coupled channels, unitarity.

\footnotetext{
${ }^{1}$ email: borasoy@itkp.uni-bonn.de

${ }^{2}$ email: rnissler@itkp.uni-bonn.de

${ }^{3}$ email: weise@ph.tum.de
} 


\section{Introduction}

Chiral perturbation theory is an appropriate framework to investigate the dynamics of hadrons at low energies, whereby symmetries and symmetry breaking patterns of QCD are incorporated. A systematic loop expansion can be carried out, but its perturbative application is often limited to a small range of energies and breaks down in the vicinity of resonances. In the $K^{-} p$ channel, for example, the existence of the $\Lambda(1405)$ resonance just below the $K^{-} p$ threshold renders $\mathrm{SU}(3)$ chiral perturbation theory (ChPT) inapplicable. However, the combination with non-perturbative coupled-channels techniques has proved useful by generating the $\Lambda(1405)$ dynamically as an $I=0 \bar{K} N$ quasibound state and as a resonance in the $\pi \Sigma$ channel [1,2].

Such approaches have been applied to a variety of meson-baryon scattering processes with quite some success [2-9]. All those calculations appear to describe the available scattering data similarly well, whereas the details of the chosen framework, e.g. the driving terms in the BetheSalpeter equation, differ in most cases. To our knowledge no attempt has so far been made to compare the different approaches systematically. To this end, we study in the present work several variants of the coupled-channels approaches to the $\bar{K} N$ system with different interaction kernels, hence providing an estimate for the model dependence of such analyses.

The $\bar{K} N$ channel is of particular interest as a testing ground for chiral $\mathrm{SU}(3)$ symmetry in QCD and for the role of explicit chiral symmetry breaking due to the relatively large strange quark mass. High-precision $K^{-} p$ threshold data set important constraints for theoretical approaches and have recently been supplemented by the new accurate results for the strong interaction shift and width of kaonic hydrogen from the DEAR experiment [10] which reduced the mean values and error ranges of the previous KEK experiment [11]. There is thus renewed interest in an improved analysis of these data along with existing information on $K^{-} p$ scattering, the $\pi \Sigma$ mass spectrum and $K^{-} p$ threshold decay ratios. Some results have already been presented in [12].

The electromagnetic interaction is responsible for the binding of kaonic hydrogen and also contributes significantly in elastic $K^{-} p$ scattering close to threshold. It must therefore be included in the investigation of the $K^{-} p$ system and we study the importance of Coulomb and isospin breaking effects in the $\bar{K} N$ system.

Another topic of interest is the pole structure of the $\Lambda(1405)$ resonance. In the context of coupled-channels approaches it has been argued that the $\Lambda(1405)$ is a superposition of two nearby poles $[5,8,13,14]$. Both poles couple with different strengths to the $\pi \Sigma$ and $\bar{K} N$ channels, so that by performing experiments with different initial states the observed peak structure should change. Photoproduction of $\Lambda(1405)$ has been studied at ELSA with the SAPHIR detector at $2.6 \mathrm{GeV}$ and in the charged decay channels, $\pi^{+} \Sigma^{-}$and $\pi^{-} \Sigma^{+}$, at SPring-8/LEPS with incident photon energies in the range $1.5<E_{\gamma}^{l a b}<2.4 \mathrm{GeV}$ [15]. Another upcoming experiment at ELSA with the Crystal Barrel detector is the decay of the $\Lambda(1405)$ into the $\pi^{0} \Sigma^{0}$ channel which provides a unique signature of $\Lambda(1405)$, since the $\pi^{0} \Sigma^{0}$ channel does not have an isospin $I=1$ component and hence does not couple to the $\Sigma(1385)$ resonance.

On the theoretical side, the positions of the relevant poles in the complex $\sqrt{s}$-plane have been studied in $[8,13]$ by using only the lowest order effective Lagrangian. We critically examine the changes in the pole positions by including the contributions from the next-to-leading order Lagrangian.

The present work is organized as follows. The effective Lagrangian and a short description of the coupled-channels method is outlined in the next section. In Sec. 3 the results of differ- 
ent coupled-channels approaches are compared with experiment and the differences between these frameworks are highlighted. Moreover, the additional tight constraints set by the new DEAR experiment are emphasized. Coulomb and isospin breaking effects are discussed. Sec. 4 summarizes our findings, while some technicalities are relegated to the appendix.

\section{Formalism}

\subsection{Effective Lagrangian}

In this section, we briefly outline the coupled-channel formalism of meson-baryon scattering. It is based on the $\mathrm{SU}(3)$ chiral effective Lagrangian which incorporates the same symmetries and symmetry breaking patterns as QCD and describes the coupling of the pseudoscalar octet $(\pi, K, \eta)$ to the ground state baryon octet $(N, \Lambda, \Sigma, \Xi)$. The Lagrangian

$$
\mathcal{L}=\mathcal{L}_{\phi}+\mathcal{L}_{\phi B}
$$

includes the mesonic term $\mathcal{L}_{\phi}$ up to second chiral order [16],

$$
\mathcal{L}_{\phi}=\frac{f^{2}}{4}\left\langle u_{\mu} u^{\mu}\right\rangle+\frac{f^{2}}{4}\left\langle\chi_{+}\right\rangle,
$$

where $\langle\ldots\rangle$ denotes the trace in flavor space. The pseudoscalar meson octet $\phi$ is arranged in a matrix valued field

$$
U(\phi)=u^{2}(\phi)=\exp \left(\sqrt{2} i \frac{\phi}{f}\right)
$$

and $f$ is the pseudoscalar decay constant in the chiral limit. The quantity $U$ enters the Lagrangian in the combinations $u_{\mu}=i u^{\dagger} \partial_{\mu} U u^{\dagger}$ and $\chi_{+}=2 B_{0}\left(u^{\dagger} \mathcal{M} u^{\dagger}+u \mathcal{M} u\right)$, the latter one involving explicit chiral symmetry breaking via the quark mass matrix $\mathcal{M}=\operatorname{diag}\left(m_{u}, m_{d}, m_{s}\right)$, and $B_{0}=-\langle 0|\bar{q} q| 0\rangle / f^{2}$ relates to the order parameter of spontaneously broken chiral symmetry.

The second piece of the Lagrangian in Eq. (1), $\mathcal{L}_{\phi B}$, describes the meson-baryon interactions and reads at lowest order $[17]$

$$
\mathcal{L}_{\phi B}^{(1)}=i\left\langle\bar{B} \gamma_{\mu}\left[D^{\mu}, B\right]\right\rangle-M_{0}\langle\bar{B} B\rangle-\frac{1}{2} D\left\langle\bar{B} \gamma_{\mu} \gamma_{5}\left\{u^{\mu}, B\right\}\right\rangle-\frac{1}{2} F\left\langle\bar{B} \gamma_{\mu} \gamma_{5}\left[u^{\mu}, B\right]\right\rangle .
$$

The $3 \times 3$ matrix $B$ collects the ground state baryon octet, $M_{0}$ is the common baryon octet mass in the chiral limit and $D, F$ denote the axial vector couplings of the baryons to the mesons. Their numerical values can be extracted from semileptonic hyperon decays and we employ the central values determined in [18]: $D=0.80, F=0.46$. The covariant derivative of the baryon field is given by

$$
\left[D_{\mu}, B\right]=\partial_{\mu} B+\left[\Gamma_{\mu}, B\right]
$$

with the chiral connection

$$
\Gamma_{\mu}=\frac{1}{2}\left[u^{\dagger}, \partial_{\mu} u\right]
$$

We also need the next-to-leading order contribution to $\mathcal{L}_{\phi B}$ which is given by

$$
\begin{aligned}
\mathcal{L}_{\phi B}^{(2)}= & b_{D}\left\langle\bar{B}\left\{\chi_{+}, B\right\}\right\rangle+b_{F}\left\langle\bar{B}\left[\chi_{+}, B\right]\right\rangle+b_{0}\langle\bar{B} B\rangle\left\langle\chi_{+}\right\rangle \\
& +d_{1}\left\langle\bar{B}\left\{u_{\mu},\left[u^{\mu}, B\right]\right\}\right\rangle+d_{2}\left\langle\bar{B}\left[u_{\mu},\left[u^{\mu}, B\right]\right]\right\rangle+d_{3}\left\langle\bar{B} u_{\mu}\right\rangle\left\langle u^{\mu} B\right\rangle+d_{4}\langle\bar{B} B\rangle\left\langle u^{\mu} u_{\mu}\right\rangle,
\end{aligned}
$$




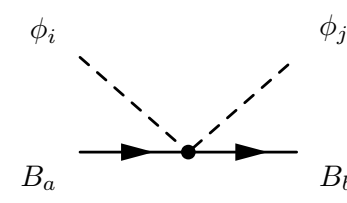

(a)

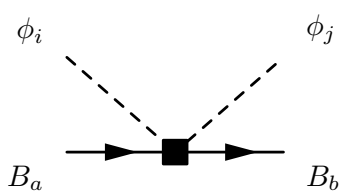

(b)

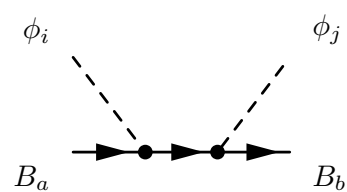

(c)

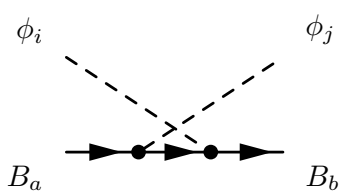

(d)

Figure 1: Shown are the $\mathcal{O}\left(p^{1}\right)$ (a) and $\mathcal{O}\left(p^{2}\right)$ (b) contact interactions as well as the direct (c) and crossed (d) Born terms. Solid and dashed lines represent baryons and pseudoscalar mesons, respectively.

where only the pieces relevant for our analysis are displayed. The values of the low-energy constants $b_{i}$ and $d_{i}$ utilized in this work have been constrained also by the recent coupledchannel analysis for $\eta$ photoproduction [7]. We will come back to this point later in Section 3 and leave their values undetermined for the moment.

\subsection{Coupled channels}

For $\bar{K} N$ scattering chiral perturbation theory based on the Lagrangian from the preceding section fails due to the presence of the nearby $\Lambda(1405)$ subthreshold resonance. Unitarity effects from final state interactions are important and must be included in a non-perturbative fashion. To this end, one computes from the Lagrangian the relativistic tree level amplitude $V_{j b, i a}\left(s, \Omega ; \sigma, \sigma^{\prime}\right)$ of the meson-baryon scattering processes $\phi_{i} B_{a}^{\sigma} \rightarrow \phi_{j} B_{b}^{\sigma^{\prime}}$ (with spin indices $\sigma$, $\left.\sigma^{\prime}\right)$. This amplitude is the driving term in the coupled-channels integral equation determining the meson-baryon T-matrix.

The effective meson-baryon Lagrangian, Eqs. $(4,7)$, has been used at different levels of sophistication in the literature. While only the Weinberg-Tomozawa term from the covariant derivative in Eq. (4) is taken, e.g., in [2], the direct and crossed Born terms are included in [5]. In [3] the Lagrangian of second chiral order is added which yields additional contact interactions, whereas in [7] the contact interactions and the direct Born term have been taken into account, but the crossed Born term has been excluded. In order to provide an estimate of the model-dependence of such approaches, we will discuss four different choices for the amplitude $V_{j b, i a}\left(s, \Omega ; \sigma, \sigma^{\prime}\right)$.

First, only the leading order contact (Weinberg-Tomozawa) term is taken into account, see Figure 1a. Subsequently, the contact interactions from the Lagrangian of second chiral order, $\mathcal{L}_{\phi B}^{(2)}$, are included, see Fig. 1b. In the third and fourth approach we add successively the direct (Fig. 1c) and crossed (Fig. 1d) Born diagrams. For brevity, we will refer to these variants as "WT" (Weinberg-Tomozawa), "c" (additional contact terms), "s" (including s-channel Born diagram) and " $u$ " (including $u$-channel Born diagram), respectively.

It turns out that already the inclusion of the next-to-leading order contact terms, which have been neglected in many previous coupled-channel analyses $[2,5,6,8]$, improves the agreement of our results with the well-measured $K^{-} p$ threshold branching ratios and the shape of the $\pi \Sigma$ mass spectrum, whereas the Born diagrams Fig. 1c,d yield only small numerical changes. The explicit expressions for the diagrams, Figs. 1a,c,d, can be found in [5], but for completeness we display the formulae of all those contributions in the appendix.

Since we are primarily concerned with a narrow center-of-mass energy region around the $\bar{K} N$ threshold, it is sufficient to restrict ourselves to the $s$-wave (matrix) amplitude $V(s)$ given 
by

$$
V(s)=\frac{1}{8 \pi} \sum_{\sigma} \int d \Omega V(s, \Omega ; \sigma, \sigma),
$$

where we have averaged over the spin $\sigma= \pm 1 / 2$ of the baryons and $s$ is the invariant energy squared. We work in the physical basis assigning each particle its physical mass. This scheme produces the correct thresholds of the different channels, and it is consistent at the order at which the driving amplitudes $V$ are calculated.

For each partial wave $l$ unitarity imposes a restriction on the (inverse) $T$-matrix above the pertinent thresholds

$$
\operatorname{Im} T_{l}^{-1}=-\frac{\left|\mathbf{q}_{c m}\right|}{8 \pi \sqrt{s}}
$$

with $\mathbf{q}_{c m}$ being the three-momentum in the center-of-mass frame of the channel under consideration. Hence the imaginary part of $T_{l}^{-1}$ is identical with the imaginary part of the basic scalar loop integral $\tilde{G}$ above threshold,

$$
\tilde{G}\left(q^{2}\right)=\int \frac{d^{d} p}{(2 \pi)^{d}} \frac{i}{\left[(q-p)^{2}-M_{B}^{2}+i \epsilon\right]\left[p^{2}-m_{\phi}^{2}+i \epsilon\right]},
$$

where $M_{B}$ and $m_{\phi}$ are the physical masses of the baryon and the meson, respectively $[5,7,19]$. In dimensional regularization we obtain for the finite part $G$ of $\tilde{G}$,

$$
\begin{aligned}
G\left(q^{2}\right)= & a(\mu)+\frac{1}{32 \pi^{2} q^{2}}\left\{q^{2}\left[\ln \left(\frac{m_{\phi}^{2}}{\mu^{2}}\right)+\ln \left(\frac{M_{B}^{2}}{\mu^{2}}\right)-2\right]\right. \\
& \left.+\left(m_{\phi}^{2}-M_{B}^{2}\right) \ln \left(\frac{m_{\phi}^{2}}{M_{B}^{2}}\right)-8 \sqrt{q^{2}}\left|\mathbf{q}_{c m}\right| \operatorname{artanh}\left(\frac{2 \sqrt{q^{2}}\left|\mathbf{q}_{c m}\right|}{\left(m_{\phi}+M_{B}\right)^{2}-q^{2}}\right)\right\},
\end{aligned}
$$

where $\mu$ is the regularization scale. The subtraction constant $a(\mu)$ cancels the scale dependence of the chiral logarithms and simulates higher order contributions with the value of $a(\mu)$ depending on the respective channel.

To the order we are working the inverse of the $T$ matrix can be written as (suppressing the subscript $l(=0)$ for brevity)

$$
T^{-1}=V^{-1}+G
$$

which yields after inversion

$$
T=[1+V \cdot G]^{-1} V .
$$

Eq. (13) is a matrix equation with the diagonal matrix $G$ collecting the loop integrals in each channel. This amounts to a summation of a bubble chain to all orders in the $s$-channel, equivalent to solving a Bethe-Salpeter equation with $V$ as driving term, where all momenta in $V$ are set to their on-shell values. This so-called on-shell scheme reduces the full Bethe-Salpeter equation to the simple matrix equation (13).

However, in the presence of the crossed Born term (Fig. 1d) this simplification must be treated with care due to the appearance of unphysical subthreshold cuts. In the unphysical region below the threshold of a given channel the propagator of the intermediate baryon in the crossed Born term leads to divergences (in the $S U(2)$ case this fact is known as the nucleon cut $[20]$ ), which correspond to logarithmic singularities in the $s$-wave amplitude. Within the coupled 
channel formalism the subthreshold cuts of heavier virtual meson-baryon pairs can contribute above the thresholds of physical processes involving lighter meson-baryon systems. Since this is an artifact of the on-shell formalism and would not be present in a full field theoretical calculation, we eliminate the unphysical subthreshold cuts by matching the contribution of the crossed Born diagram to a constant value below a certain invariant energy $\sqrt{s_{0}}$. We have convinced ourselves that our conclusions do not depend on the specific choice of $\sqrt{s_{0}}$ as long as it is not too close to the singularities. As a matter of fact, the contribution of the crossed Born diagram to the full $s$-wave interaction kernel $V$ turns out to be numerically small.

\subsection{Coulomb interaction}

The Coulomb interaction has been shown to yield significant contributions to the elastic $K^{-} p$ scattering amplitude up to kaon laboratory momenta of $100-150 \mathrm{MeV} / c$ [21]. Close to $K^{-} p$ threshold the electromagnetic interactions are thus important as well and should not be neglected as in previous coupled channel calculations $[2,3,5,6,8]$. The quantum-mechanical Coulomb scattering amplitude for point charges can be calculated exactly and reads [22]

$$
f_{K^{-} p \rightarrow K^{-} p}^{\text {coul }}=\frac{1}{2 \mathbf{q}_{c m}^{2} a_{B} \sin ^{2}\left(\theta_{c m} / 2\right)} \frac{\Gamma\left(1-i /\left(\left|\mathbf{q}_{c m}\right| a_{B}\right)\right)}{\Gamma\left(1+i /\left(\left|\mathbf{q}_{c m}\right| a_{B}\right)\right)} \exp \left[\frac{2 i}{\left|\mathbf{q}_{c m}\right| a_{B}} \ln \sin \frac{\theta_{c m}}{2}\right],
$$

where $a_{B}=84 \mathrm{fm}$ is the Bohr radius of the $K^{-} p$ system, while $\mathbf{q}_{\mathrm{cm}}$ and $\theta_{\mathrm{cm}}$ denote the center-ofmass three-momentum and scattering angle, respectively. We account for the electromagnetic interaction by adding $f_{K^{-} p \rightarrow K^{-} p}^{\text {coul }}$ to the unitarized strong elastic $K^{-} p$ amplitude

$$
f_{K^{-} p \rightarrow K^{-} p}^{\mathrm{str}}=\frac{1}{8 \pi \sqrt{s}} T_{K^{-} p \rightarrow K^{-} p}^{\mathrm{str}}
$$

The total elastic cross section is then obtained by performing the integration of $d \sigma / d \Omega=$ $\left|f^{\text {coul }}+f^{\text {str }}\right|^{2}$ over the center-of-mass scattering angle. Since this expression is divergent for forward scattering, a cutoff for the scattering angle must be introduced. In the analysis of the scattering data [23,24], forward angles were suppressed by accepting only events with $\theta_{c m}$ larger than a minimum angle $\theta_{\text {min }}$. In practice the value employed in ref. [23, 24] was $\cos \theta_{\min }=0.966$. We choose the same $\theta_{\min }$ for a meaningful comparison with data. Some $K^{-} p$ angular distributions (though of very limited quality) were reported in ref. [23]. We have checked that our treatment of Coulomb effects reproduces the measured small-angle differential cross sections in the relevant momentum range. The dependence of our results on the infrared cutoff provided by $\theta_{\min }$ will be discussed in the Section 3.4.

The Coulomb potential vanishes at infinity as $1 / r$ and leads to an infrared divergent scattering amplitude for $\mathbf{q}_{c m} \rightarrow 0$. In physical reality, however, the kaons are scattered off neutral hydrogen atoms rather than off protons and the range of the Coulomb interaction - given by the Bohr radius of the hydrogen atom - is therefore finite. Deviations from the pure Coulomb potential will be important, if the de Broglie wavelength of the kaons is of the order of the atomic radius, corresponding to kaon laboratory momenta of a few keV/c. The lowest experimentally accessible kaon momenta are around $100 \mathrm{MeV} / c$, four orders of magnitude higher, so the electronic shielding of the Coulomb potential can be safely neglected.

Deviations from the point Coulomb scattering amplitude are expected when the wavelength of the incident kaon is comparable to the size of the proton. This translates into kaon momenta 
larger than $200 \mathrm{MeV} / \mathrm{c}$. For such momenta $K^{-} p$ scattering is completely dominated by the strong interaction since the Coulomb amplitude decreases as $1 / \mathbf{q}_{\mathrm{cm}}^{2}$. The corrections induced by finite size effects in the Coulomb amplitude are negligible in the relevant range of kaon energies. We will therefore work with the formula given in Eq. (14) combined with the small-angle cut as mentioned before.

\section{Results and Discussion}

In this section we present and discuss the numerical results of our calculation. Low-energy antikaon-nucleon scattering and reactions have been studied experimentally decades ago [23-28]. The available data (admittedly with large errors) are mostly restricted to $K^{-}$momenta above $100 \mathrm{MeV} / \mathrm{c}$. On the other hand, there is the new and precise DEAR measurement of the strong interaction shift and width in kaonic hydrogen [10] as well as a similar recent analysis of the KEK collaboration [11], which set constraints for the strong-interaction part of the elastic $K^{-} p$ amplitude at threshold. Further tight constraints are imposed by the accurately determined threshold branching ratios into the inelastic channels $\pi \Sigma$ and $\pi^{0} \Lambda$ [29,30]:

$$
\begin{aligned}
\gamma & =\frac{\Gamma\left(K^{-} p \rightarrow \pi^{+} \Sigma^{-}\right)}{\Gamma\left(K^{-} p \rightarrow \pi^{-} \Sigma^{+}\right)}=2.36 \pm 0.04 \\
R_{c} & =\frac{\Gamma\left(K^{-} p \rightarrow \pi^{+} \Sigma^{-}, \pi^{-} \Sigma^{+}\right)}{\Gamma\left(K^{-} p \rightarrow \text { all inelastic channels }\right)}=0.664 \pm 0.011 \\
R_{n} & =\frac{\Gamma\left(K^{-} p \rightarrow \pi^{0} \Lambda\right)}{\Gamma\left(K^{-} p \rightarrow \text { neutral states }\right)}=0.189 \pm 0.015
\end{aligned}
$$

and by the $\pi \Sigma$ invariant mass spectrum in the isospin $I=0$ channel [31].

Our approach has six subtractions constants $a(\mu)$ in the different channels and eight constants given within certain limited ranges: the decay constant $f$ and the higher order couplings $b_{i}, d_{i}$. As mentioned before, the $b_{i}$ and $d_{i}$ have been constrained by the analysis of [7] which includes $\eta$ photoproduction as a high quality data set. Since both pions and kaons are involved we choose to vary the decay constant $f$ in the range given by its value in the chiral limit, $f=88 \mathrm{MeV}[32]$, and the physical kaon decay constant $F_{K}=112.7 \mathrm{MeV}$. Furthermore, only the " $s$ " approach in our investigation, i.e. the one that involves the leading and next-to-leading order contact interactions as well as the direct Born term, exactly coincides with the framework chosen in [7]. We can therefore expect moderate deviations in the numerical determination of the coupling constants from a fit to low-energy hadronic data.

In the first part of this section, we compare the four different approaches described in the preceding section which follow from the successive inclusion of the diagrams in Fig. 1 in the interaction kernel $V$. It turns out that in all four cases the results cannot be brought into simultaneous satisfactory agreement with the elastic $K^{-} p$ elastic cross section and the kaonic hydrogen data from the DEAR experiment [10], although the inclusion of the Coulomb interaction ameliorates the situation compared to previous coupled-channel calculations. In order to examine how well the four approaches under consideration agree with the scattering data, we first exclude the DEAR data from the fit and "predict" the strong-interaction shift and width in kaonic hydrogen based on the rest of the low-energy scattering data. We note that the " $u$ " approach yields the fit with the smallest overall $\chi^{2}$ value, but only slightly larger 
values are obtained in the "c" and " $s$ " versions, whereas the "WT" model, based only on the leading Weinberg-Tomozawa term, has a significantly larger $\chi^{2}$. However, one should keep in mind that the "WT" approach has less parameters.

As a second step, we then investigate the changes of the results when the DEAR data are included in the fit. For this purpose it is sufficient to restrict ourselves to the " $u$ " ansatz involving all the diagrams in Fig. 1-i.e. the entire set of next-to-leading order contributions to the $s$-wave amplitude - since qualitatively similar results are obtained in the " $\mathrm{c}$ " and " $s$ " models.

The third part of this section is devoted to the detailed discussion of Coulomb corrections in the elastic $K^{-} p$ scattering cross section, and we conclude with a study of the relevant resonance poles in the complex energy plane.

\subsection{Comparison of the different approaches}

We have first performed an overall $\chi^{2}$ fit to the available low energy $\bar{K} N$ data excluding the strong level shift and width of kaonic hydrogen. In order to emphasize the importance of the precisely measured threshold branching ratios, the $\chi^{2}$ value of each observable has been divided by the number of pertinent data points. The resulting numerical values of the parameters are compiled in Table 1 . We point out that the subtraction constants $a(\mu)$ translate into values close to -2 in the framework of [5] and are therefore - according to the authors - "of natural size". Our subtraction constants in the important channels $\pi \Sigma, \bar{K} N$ are also roughly compatible with the numbers following from the matching condition to the crossed amplitude as advocated in [6]. In the "WT" and "c" approaches, the value of the pseudoscalar decay constant $f$ tends towards the physical kaon decay constant, whereas it is lowered by the inclusion of $s$ - and $u$ channel Born terms. The low energy constants $b_{i}, d_{i}$ are roughly compatible with the numbers obtained from $\eta$ photoproduction by employing a closely related coupled channel approach [7]. We note that the $b_{i}$ parameters in the " $\mathrm{u}$ " fit correspond at tree level to the $K N$ sigma terms $\sigma_{K N}^{(1)}(0)=305 \mathrm{MeV}$ and $\sigma_{K N}^{(2)}(0)=181 \mathrm{MeV}$. These numbers are in fair agreement with the values for the tree level contributions presented in [33] (including a $\pi N$ sigma term $\sigma_{\pi N}(0) \simeq 30$ $\mathrm{MeV}$ at tree level).

In Fig. 2 we show the results for the elastic and inelastic cross sections of $K^{-} p$ scattering. The four lines correspond to the four different approaches under consideration, all of them in good agreement with the experimental data. The $\pi \Sigma$ mass spectrum in the isospin $I=0$ channel is displayed in Fig. 3a. It is well reproduced by the approaches which include the additional $\mathcal{O}\left(p^{2}\right)$ contact terms ("c", "s", " $u$ "), whereas the "WT" approach fails to explain the experimental data points for higher invariant energies. Following ref. [5] the experimental data displayed in Fig. 3 can be regarded as a $\pi^{-} \Sigma^{+}$event distribution originating from a generic $s$-wave $I=0$ source. This source is assumed to be dominated by $\bar{K} N$ and $\pi \Sigma I=0$ states which are multiplied by energy-independent coefficients $r_{1}$ and $r_{2}$, respectively. Since the experimental data are not normalized, only the ratio $r_{1} / r_{2}$ is of significance. Utilizing this ansatz, we obtain the curves in Fig. $3 \mathrm{~b}$ and observe that now all four approaches reproduce the experimental spectrum almost equally well. But the number of free parameters has been increased by one and - consequently - the quality of the fits has improved. The ratios $r_{1} / r_{2}$ range between 1.40 for the "WT" and 0.96 for the "c" approach. Within this scenario an $I=0$ source with roughly equal portions of initial $\bar{K} N$ and $\pi \Sigma$ states thus seems to be favored by all schemes, with a tendency toward higher $\bar{K} N$ shares. 
The results for the threshold branching ratios are compiled in Table 2. Independently of the chosen approach, the quantities $\gamma$ and $R_{n}$ agree well with the experimental numbers. For the branching ratio $R_{c}$ into charged final states the situation is different. Whereas the "c", " $s$ ", and " $u$ " fits are in perfect agreement with the experimental error bars, the "WT" result happens to be too small in magnitude emphasizing again the importance of $\mathcal{O}\left(p^{2}\right)$ contact terms.

Having so far omitted the strong interaction shift and width in kaonic hydrogen from the fits, we can now predict these observables for the different approaches. To this end we employ the result of [35] relating the ground state strong energy shift $\Delta E$ and width $\Gamma$ of kaonic hydrogen to the $K^{-} p$ scattering length $a_{K^{-}}$in the presence of electromagnetic corrections:

$$
\Delta E-\frac{i}{2} \Gamma=-2 \alpha^{3} \mu_{c}^{2} a_{K^{-} p}\left[1-2 \alpha \mu_{c}(\ln \alpha-1) a_{K^{-} p}\right] .
$$

The reduced mass of the $K^{-} p$ system is denoted by $\mu_{c}, \alpha$ is the fine-structure constant, and the scattering length $a_{K^{-}}$is given by the strong interaction $T$ matrix at threshold

$$
a_{K^{-} p}=\left.\frac{1}{8 \pi \sqrt{s}} T_{K^{-} p \rightarrow K^{-} p}(s)\right|_{s=\left(m_{K^{-}}+M_{p}\right)^{2}} .
$$

In order to demonstrate the importance of the electromagnetic corrections calculated in [35], we compare Eq. (17) with the predictions derived from the well-known Deser-Trueman formula [36]

$$
\Delta E-\frac{i}{2} \Gamma=-2 \alpha^{3} \mu_{c}^{2} a_{K^{-} p}
$$

and the kaonic hydrogen data from the DEAR [10] and KEK [11] experiments in Fig. 4; the pertinent numerical values are displayed in Table 3. The shifts and widths corresponding to the different approaches agree all with the error ranges given in [11] if Eq. (17) is utilized. In contrast, both the shift and the width of the new DEAR experiment cannot be accommodated by the coupled-channels approaches constrained by scattering and reaction cross sections, although the electromagnetic corrections given in [35] reduce the width $\Gamma$ by a significant amount. As can be seen in Fig. 4 the disagreement is reduced by the inclusion of higher order contact terms (approaches "c", " $s$ ", " $u$ ").

In summary we note that the approaches which include the $\mathcal{O}\left(p^{2}\right)$ contact terms ("c", " $s$ ", " $u$ ") describe all available low energy hadronic scattering data excluding kaonic hydrogen experiments at DEAR. The fits to the $K^{-} p \rightarrow \pi^{-} \Sigma^{+}$cross section and the (related) branching ratio $R_{c}$ which we obtain within the "WT" approach are not of the same high quality. In this case a decent description of the $\pi \Sigma$ mass spectrum in Fig. 3 can only be achieved by utilizing the ad-hoc parametrization suggested in [5], but not by a simple isospin zero $\pi \Sigma$ invariant mass distribution. One should keep in mind however that the leading order "WT" framework is oversimplified. It does not involve the coupling constants $b_{i}, d_{i}$ which turn out to be important in the more complete approaches.

\subsection{Inclusion of the DEAR data}

As already mentioned, the new high-precision DEAR data [10] set additional tight constraints on $\bar{K} N$ interactions. In this section we explore changes of our results when the DEAR data are included in the fit. For brevity we restrict ourselves to the discussion of the " $u$ " scheme, the one 


\begin{tabular}{|ll|c|c|c|c|}
\hline & & "WT" & "c" & "s" & " $u "$ \\
\hline$a_{\bar{K} N}$ & $\left(10^{-3}\right)$ & -0.38 & -1.64 & -2.13 & -2.16 \\
\hline$a_{\pi \Lambda}$ & $\left(10^{-3}\right)$ & 0.21 & 5.43 & -2.32 & -6.34 \\
\hline$a_{\pi \Sigma}$ & $\left(10^{-3}\right)$ & 2.69 & -1.01 & -2.16 & -1.24 \\
\hline$a_{\eta \Lambda}$ & $\left(10^{-3}\right)$ & 4.73 & 2.36 & -0.53 & -1.99 \\
\hline$a_{\eta \Sigma}$ & $\left(10^{-3}\right)$ & 5.56 & 1.72 & 3.55 & -2.75 \\
\hline$a_{K \Xi}$ & $\left(10^{-3}\right)$ & -4.38 & 2.91 & 0.32 & -4.37 \\
\hline$f$ & $\left(\mathrm{MeV}^{-1}\right)$ & 111.2 & 111.6 & 103.6 & 103.3 \\
\hline$b_{0}$ & $\left(\mathrm{GeV}^{-1}\right)$ & - & -0.24 & -0.27 & -0.31 \\
\hline$b_{D}$ & $\left(\mathrm{GeV}^{-1}\right)$ & - & 0.03 & 0.00 & 0.00 \\
\hline$b_{F}$ & $\left(\mathrm{GeV}^{-1}\right)$ & - & -0.02 & -0.12 & -0.13 \\
\hline$d_{1}$ & $\left(\mathrm{GeV}^{-1}\right)$ & - & -0.15 & -0.15 & -0.16 \\
\hline$d_{2}$ & $\left(\mathrm{GeV}^{-1}\right)$ & - & 0.11 & 0.11 & 0.12 \\
\hline$d_{3}$ & $\left(\mathrm{GeV}^{-1}\right)$ & - & 0.28 & 0.31 & 0.25 \\
\hline$d_{4}$ & $\left(\mathrm{GeV}^{-1}\right)$ & - & -0.32 & -0.31 & -0.23 \\
\hline
\end{tabular}

Table 1: Numerical values of the parameters for the different approaches. The subtraction constants are taken at $\mu=1 \mathrm{GeV}$.

\begin{tabular}{|c|c|c|c|c||c|}
\hline & "WT" & "c" & "s" & " $u$ " & Exp. $[29,30]$ \\
\hline$\gamma$ & 2.35 & 2.36 & 2.36 & 2.36 & $2.36 \pm 0.04$ \\
\hline$R_{c}$ & 0.635 & 0.655 & 0.655 & 0.661 & $0.664 \pm 0.011$ \\
\hline$R_{n}$ & 0.203 & 0.189 & 0.187 & 0.189 & $0.189 \pm 0.015$ \\
\hline
\end{tabular}

Table 2: Threshold branching ratios as defined in the text, resulting from the different approaches.

\begin{tabular}{|ll|c|c|c|c|}
\hline & & "WT" & "c" & " $s "$ & " $u "$ \\
\hline$a_{K^{-} p}$ & $(\mathrm{fm})$ & $-0.83+1.10 i$ & $-0.75+0.86 i$ & $-0.85+0.84 i$ & $-0.78+0.92 i$ \\
\hline$\Delta E_{D}$ & $(\mathrm{eV})$ & 344 & 311 & 350 & 321 \\
\hline$\Gamma_{D}$ & $(\mathrm{eV})$ & 904 & 712 & 692 & 755 \\
\hline$\Delta E_{c}(\mathrm{eV})$ & 374 & 321 & 349 & 335 \\
\hline$\Gamma_{c}(\mathrm{eV})$ & 691 & 560 & 526 & 589 \\
\hline
\end{tabular}

Table 3: Shown are the $K^{-} p$ scattering lengths $a_{K^{-}}$as well as the strong interaction shift $\Delta E$ and width $\Gamma$ in kaonic hydrogen resulting from Eq. (19) (subscript $D$ ) and Eq. (17) (subscript $c)$. 

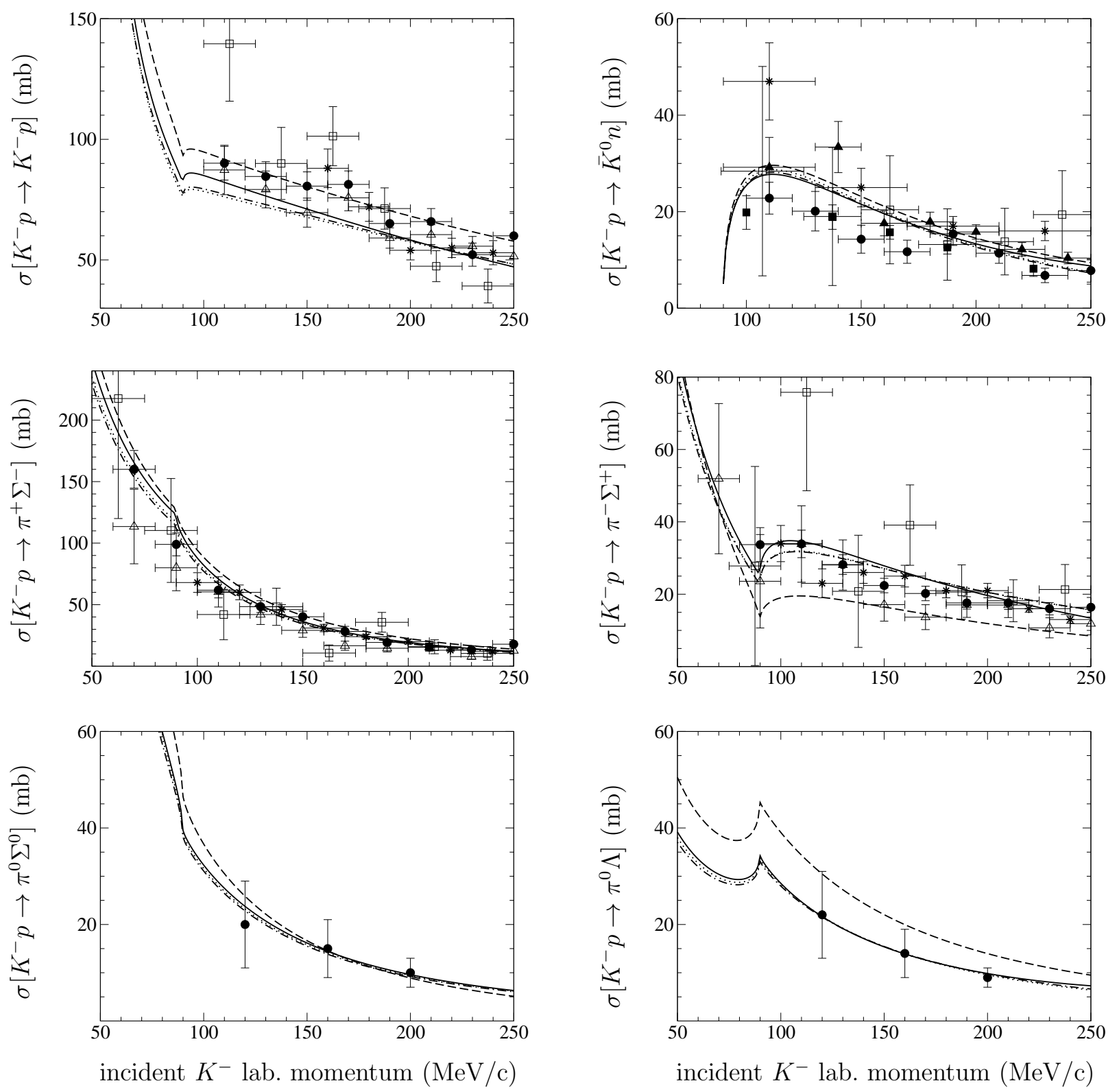

Figure 2: Total cross sections for $K^{-} p$ scattering into various channels. The data are taken from [23] (empty squares), [24] (empty triangles), [25] (filled circles), [26] (filled squares), [27] (filled triangles), [28] (stars). The dashed, dotted, dot-dashed and solid lines correspond to the approaches "WT", "c", "s" and " $u$ ", respectively. 


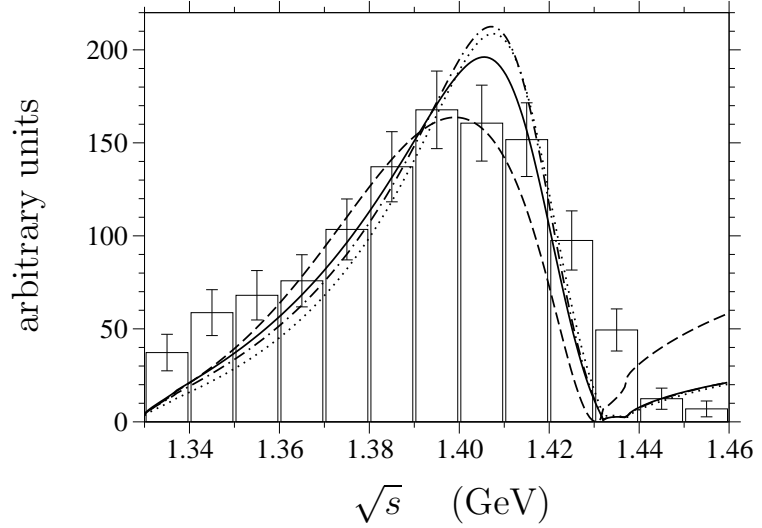

(a)

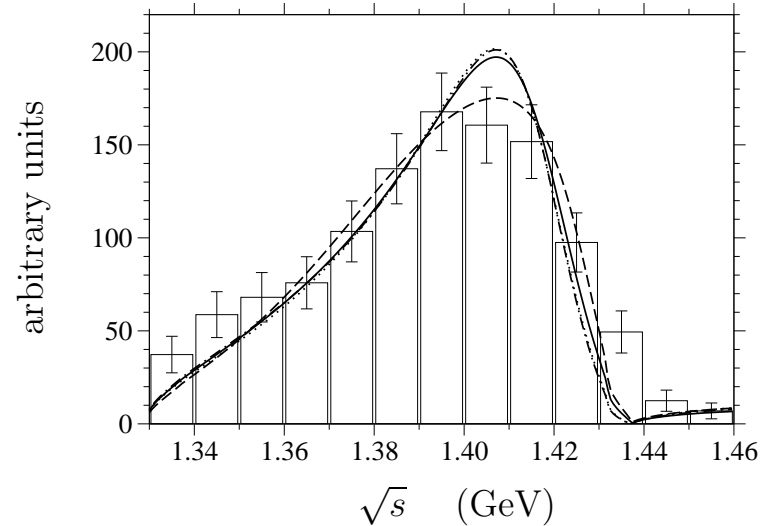

(b)

Figure 3: $\pi^{-} \Sigma^{+}$event distribution from [31], where statistical errors have been supplemented following [34]. The curves in diagram (a) where obtained by assuming a $\pi \Sigma$ invariant mass spectrum with $I=0$; the curves in diagram (b) result from the ansatz advocated in [5]. The dashed, dotted, dot-dashed and solid lines correspond to the "WT", "c", "s" and "u" approach, respectively.

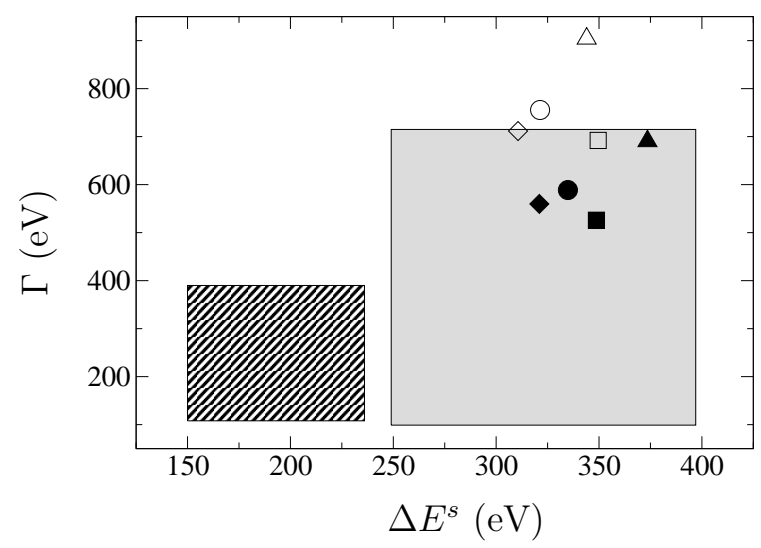

Figure 4: Predictions for the strong interaction shift and width of kaonic hydrogen from the different approaches both by using the Deser-Trueman formula, Eq. (19), (empty symbols) and by including isospin breaking corrections, Eq. (17), (filled symbols). The "WT", "c", "s" and " $u$ " approach is depicted by triangles, diamonds, squares and circles, respectively. The DEAR data are represented by the shaded box [10], the KEK data by the light gray box [11]. 
that has turned out most successful in the previous steps. Apart from yielding the least overall $\chi^{2}$ fit in the previous section, it includes the full set of next-to-leading order contributions to the interaction kernel. We utilize this " $u$ " fit and rename it "1". Forcing the fit to strictly remain within the error range given by the DEAR experiment we obtain result "3", see Fig. 5 . The detailed numbers can be found in Table 4. Fit "2" represents a compromise between fits " 1 " and " 3 " in the presence of the DEAR data. (Note, however, that fit " 2 " is not unique; and is presented here only to illustrate the changes seen in $K^{-} p$ scattering processes when the DEAR data are approached from the initial fit " 1 ".) The numerical values of the parameters for the different fits are collected in Table 5.

Total cross sections of $K^{-} p$ scattering into various channels are shown in Fig. 6. Deviations between fit "3" (which satisfies the DEAR constraints) and fit " 1 " (where these constraints have been omitted) are most pronounced in the elastic channel $K^{-} p \rightarrow K^{-} p$. Approaching the DEAR data by going from fit " 1 " to fit " 2 " and eventually to fit " 3 " subsequently lowers the total elastic cross section in the whole energy range under consideration and produces results which lie below the experimental data points (not without mentioning again that these data sets themselves scatter over a wide range). While there may be questions about the detailed treatment of Coulomb corrections, these effects can safely be neglected for kaon momenta above $200 \mathrm{MeV} / c$. Our findings suggest that within coupled-channels schemes constrained by large amounts of data, the new accurate DEAR results and the old elastic $K^{-} p$ scattering cross sections at low energy cannot be simultaneously accommodated.

The results in the inelastic $K^{-} p$ scattering channels are not altered significantly by the inclusion of the DEAR data, with the exception of the reaction $K^{-} p \rightarrow \pi^{ \pm} \Sigma^{\mp}$ where the cross sections resulting from fit " 3 " are slightly reduced. However, the threshold values of these curves also enter the branching ratios $\gamma$ and $R_{c}$. While $\gamma$, i.e. the branching ratio of the two charged $\pi \Sigma$ channels, remains within experimental errors, cf. Table 6 , the value of $R_{c}$ drops substantially below the experimental boundary when moving from fit " 1 " to fit " 3 ". This fact raises another consistency issue. While the elastic $K^{-} p$ scattering data close to threshold include the Coulomb interaction, both the branching ratio $R_{c}$ and the observables measured at DEAR represent exclusively effects of the strong interaction. These observables therefore provide a cleaner consistency check than elastic $K^{-} p$ scattering. The branching ratio $R_{n}$, on the other hand, involves neutral channels. It turns out to be uncritical and it is well reproduced by any of the fits.

If the $\pi^{-} \Sigma^{+}$event distribution from [31] is interpreted as a pure $I=0 \pi \Sigma$ invariant mass spectrum, approaching the DEAR data results in shifting the peak of the curve to lower energies and therefore worsening the fit to the data, cf. Fig. 7a. The assumption of a generic $I=0$ source made up of an admixture of $\bar{K} N$ and $\pi \Sigma$ states [5] improves the fit by introducing one additional parameter, see Fig. $7 \mathrm{~b}$. One striking feature is that the resulting ratio $r_{1} / r_{2}=2.23$ for fit "3" deviates substantially from those fits for which the DEAR data were not taken into account, and corresponds to a source that is dominated by $\bar{K} N$ states also below threshold.

As pointed out in [37] $\Lambda(1405)$ photoproduction, which has been investigated experimentally at SPring-8 [15] and at ELSA, could serve as a tool to constrain $\bar{K} N$ dynamics below threshold. If $t$-channel exchange of $K^{-}$mesons can be isolated, it should be possible to extract from the process $\gamma p \rightarrow K^{+} \pi \Sigma$ the $K^{-} p \rightarrow \pi \Sigma$ amplitudes below the $K^{-} p$ threshold. This statement is also of interest for the present investigation, since the fits which either in- or exclude the DEAR data yield different predictions for these amplitudes. In Fig. 8 we plot the quantity $4\left|\mathbf{q}_{c m}^{K^{-} p}\right| \sqrt{s} \sigma_{K^{-} p \rightarrow \pi^{\mp} \Sigma^{ \pm}}(s)$ continued below threshold, and the experimental cross section data 


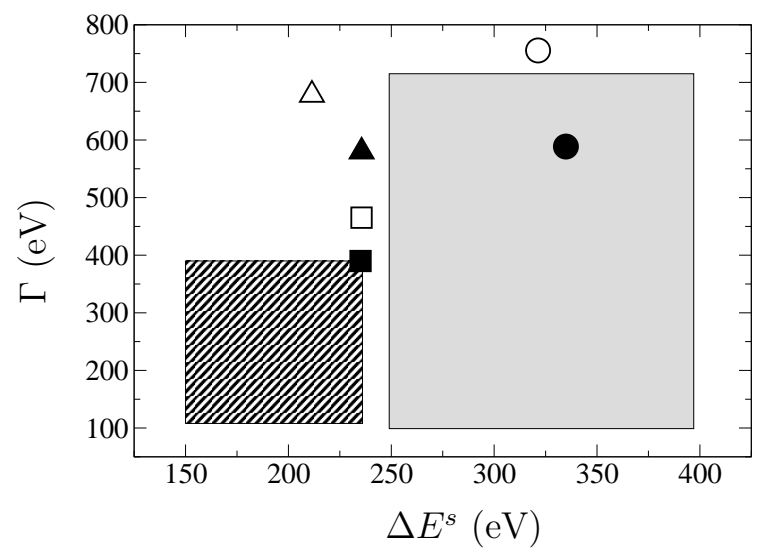

Figure 5: Results for the strong interaction shift and width of kaonic hydrogen from the fits "1", "2" and "3" depicted by circles, triangles and squares. Empty symbols correspond to the Deser-Trueman formula, Eq. (19), full symbols to Eq. (17), where isospin breaking corrections are included. The DEAR data are represented by the shaded box [10], the KEK data by the light gray box [11].

\begin{tabular}{|lc|c|c|c|}
\hline & & "1" & "2" & "3" \\
\hline$a_{K-p}$ & $(\mathrm{fm})$ & $-0.78+0.92 i$ & $-0.51+0.82 i$ & $-0.57+0.56 i$ \\
\hline$\Delta E_{D}$ & $(\mathrm{eV})$ & 321 & 211 & 236 \\
\hline$\Gamma_{D}$ & $(\mathrm{eV})$ & 755 & 678 & 465 \\
\hline$\Delta E_{c}$ & $(\mathrm{eV})$ & 335 & 236 & 235 \\
\hline$\Gamma_{c}$ & $(\mathrm{eV})$ & 589 & 580 & 390 \\
\hline
\end{tabular}

Table 4: Shown are the $K^{-} p$ scattering lengths $a_{K^{-}}$as well as the strong interaction shift $\Delta E$ and width $\Gamma$ in kaonic hydrogen resulting from Eq. (19) (subscript D) and Eq. (17) (subscript $c)$.

above the $K^{-} p$ threshold have been normalized accordingly. In the case of fit "3", the one consistent with the DEAR data, the shape of the curve is altered for both final states $\pi^{-} \Sigma^{+}$ and $\pi^{+} \Sigma^{-}$. Compared to fit "1" the peak position is shifted to lower energies, while the width is considerably increased. This difference can be examined experimentally once the necessary $t$-channel analysis and normalization of the SPring- 8 results [15] has been performed. These data cover an energy range from the $\pi \Sigma$ threshold up to energies above the $K^{-} p$ threshold, where consistency with existing cross section data can be tested. In conclusion, the SPring8/ELSA experiments may provide a further important consistency check of scattering data and the DEAR results within our framework.

It is instructive to investigate the real and imaginary parts of the elastic $K^{-} p \rightarrow K^{-} p$ scattering amplitude below threshold (see Figs. 9, 10). The important role of next-to-leading order dynamics (the "c", " $s$ " and " $u$ " versions) as compared to the leading order driven only by the Weinberg-Tomozawa term (the "WT" version) becomes visible in Fig. 9. The influence of the additional constraint imposed by the DEAR threshold data is seen in Fig. 10. It has a pronounced effect in shifting the $\Lambda(1405)$ resonance spectrum further down in $\sqrt{s}$, primarily by enforcing a smaller imaginary part of $f_{K^{-} p \rightarrow K^{-} p}$ at threshold. 

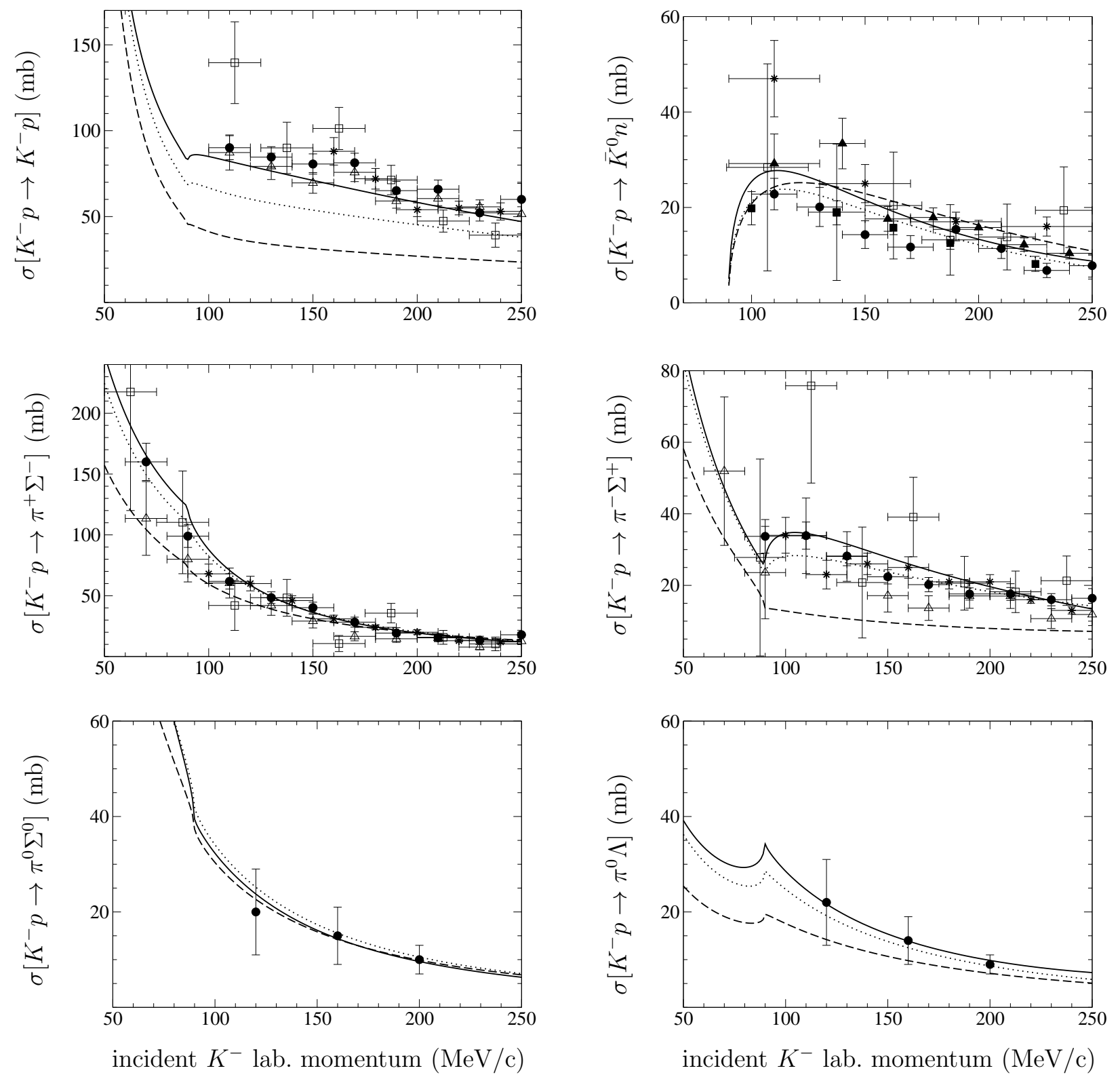

Figure 6: Total cross sections of $K^{-} p$ scattering into various channels. The data are taken from [23] (empty squares), [24] (empty triangles), [25] (filled circles), [26] (filled squares), [27] (filled triangles), [28] (stars). The solid, dotted and dashed lines represent the fits "1", "2" and "3", respectively. 


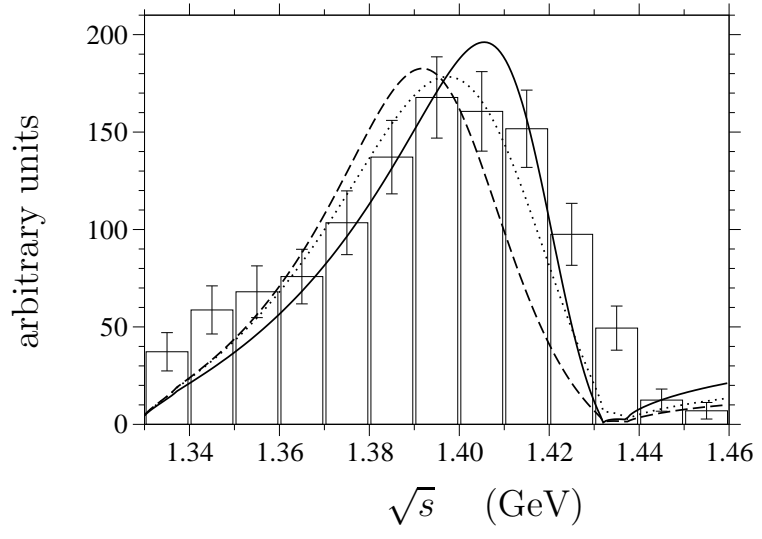

(a)

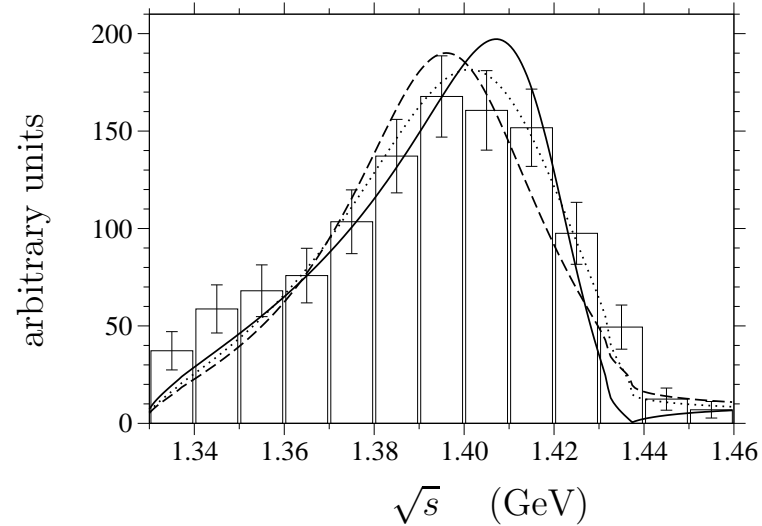

(b)

Figure 7: $\pi^{-} \Sigma^{+}$event distribution from [31], where statistical errors have been supplemented following [34]. The curves in diagram (a) where obtained by assuming a $\pi \Sigma$ invariant mass spectrum with $I=0$; the curves in diagram (b) result from the ansatz advocated in [5]. The solid, dotted and dashed lines correspond to the fits "1", "2" and "3", respectively.

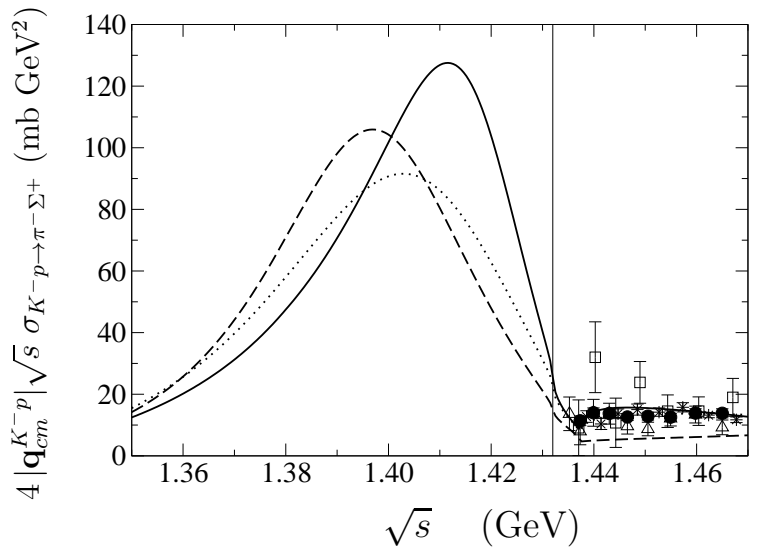

(a)

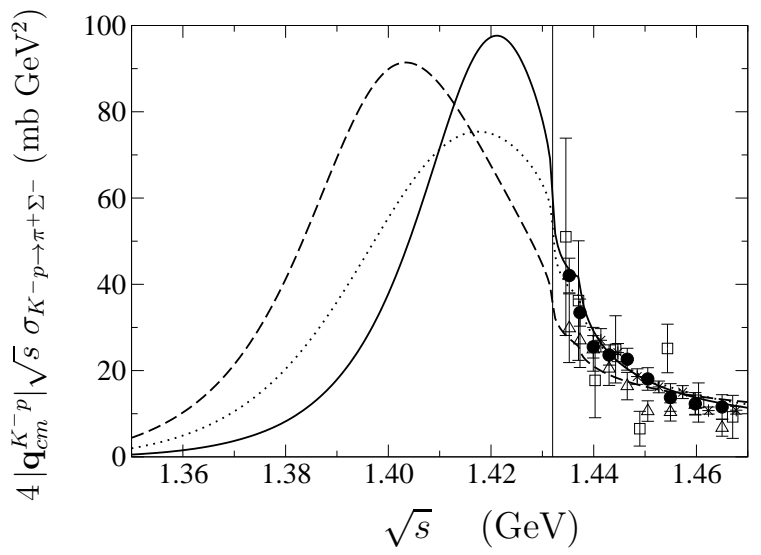

(b)

Figure 8: Shown are the cross sections for $K^{-} p \rightarrow \pi^{-} \Sigma^{+}$(a) and $K^{-} p \rightarrow \pi^{+} \Sigma^{-}$(b) multiplied by $4\left|\mathbf{q}_{c m}^{K^{-} p}\right| \sqrt{s}$ and continued below $K^{-} p$ threshold (vertical line). The experimental data points are the same as in Fig. 6, but have been modified accordingly. The solid, dotted and dashed lines correspond to the fits " 1 ", "2" and "3", respectively. 


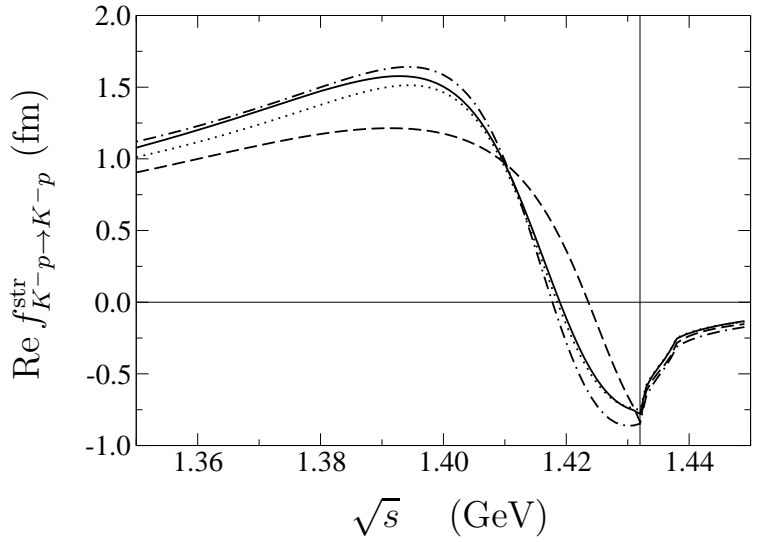

(a)

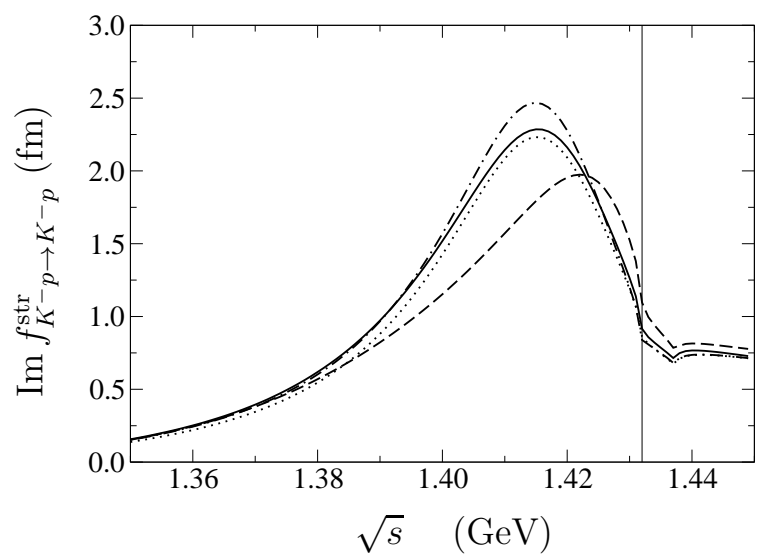

(b)

Figure 9: Real (left panel) and imaginary part (right panel) of the strong interaction elastic $K^{-} p$ amplitude. The dashed, dotted, dot-dashed and solid lines correspond to the approaches "WT", "c", " $s$ " and " $u$ ", respectively. The $K^{-} p$ threshold is indicated by the vertical line.

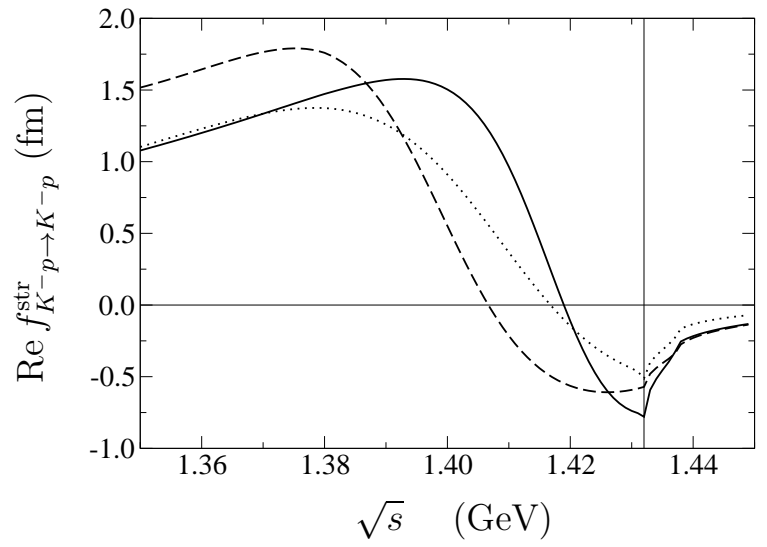

(a)

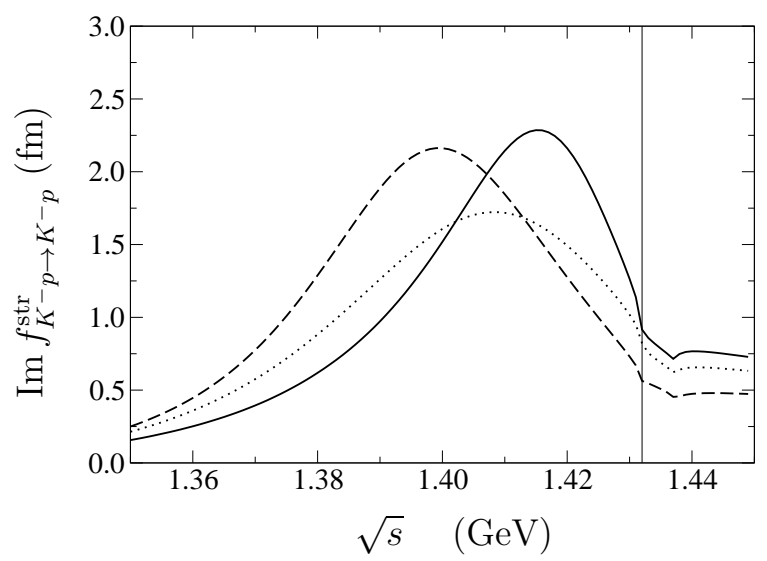

(b)

Figure 10: Real (left panel) and imaginary part (right panel) of the strong interaction elastic $K^{-} p$ amplitude. The solid, dotted and dashed lines represent the fits " 1 ", "2" and " 3 ", respectively. The $K^{-} p$ threshold is indicated by the vertical line. 


\begin{tabular}{|c|c|c|c|c|}
\hline & & "1" & "2" & "3" \\
\hline$a_{\bar{K} N}$ & $\left(10^{-3}\right)$ & -2.16 & -0.95 & -2.62 \\
\hline$a_{\pi \Lambda}$ & $\left(10^{-3}\right)$ & -6.34 & 0.59 & 11.46 \\
\hline$a_{\pi \Sigma}$ & $\left(10^{-3}\right)$ & -1.24 & -1.80 & -3.06 \\
\hline$a_{\eta \Lambda}$ & $\left(10^{-3}\right)$ & -1.99 & -2.92 & 5.10 \\
\hline$\overline{a_{\eta \Sigma}}$ & $\left(10^{-3}\right)$ & -2.75 & -0.98 & -4.26 \\
\hline$a_{K \Xi}$ & $\left(10^{-3}\right)$ & -4.37 & -2.90 & 3.69 \\
\hline$f$ & $(\mathrm{MeV})$ & 103.3 & 103.1 & 94.4 \\
\hline$b_{0}$ & $\left(\mathrm{GeV}^{-1}\right)$ & -0.31 & -0.36 & -0.20 \\
\hline$b_{D}$ & $\left(\mathrm{GeV}^{-1}\right)$ & 0.00 & 0.00 & 0.14 \\
\hline$b_{F}$ & $\left(\mathrm{GeV}^{-1}\right)$ & -0.13 & -0.13 & -0.11 \\
\hline$d_{1}$ & $\left(\mathrm{GeV}^{-1}\right)$ & -0.16 & -0.11 & -0.30 \\
\hline$d_{2}$ & $\left(\mathrm{GeV}^{-1}\right)$ & 0.12 & 0.05 & 0.02 \\
\hline$d_{3}$ & $\left(\mathrm{GeV}^{-1}\right)$ & 0.25 & 0.31 & 0.39 \\
\hline$d_{4}$ & $\left(\mathrm{GeV}^{-1}\right)$ & -0.23 & -0.32 & -0.35 \\
\hline
\end{tabular}

Table 5: Numerical values of the parameters for the different fits described in the text. The subtraction constants are taken at $\mu=1 \mathrm{GeV}$.

\begin{tabular}{|c|c|c|c||c|}
\hline & "1" & "2" & "3" & Exp. $[29,30]$ \\
\hline$\gamma$ & 2.36 & 2.35 & 2.38 & $2.36 \pm 0.04$ \\
\hline$R_{c}$ & 0.661 & 0.653 & 0.631 & $0.664 \pm 0.011$ \\
\hline$R_{n}$ & 0.189 & 0.194 & 0.176 & $0.189 \pm 0.015$ \\
\hline
\end{tabular}

Table 6: Threshold branching ratios as defined in the text, resulting from the different fits. 


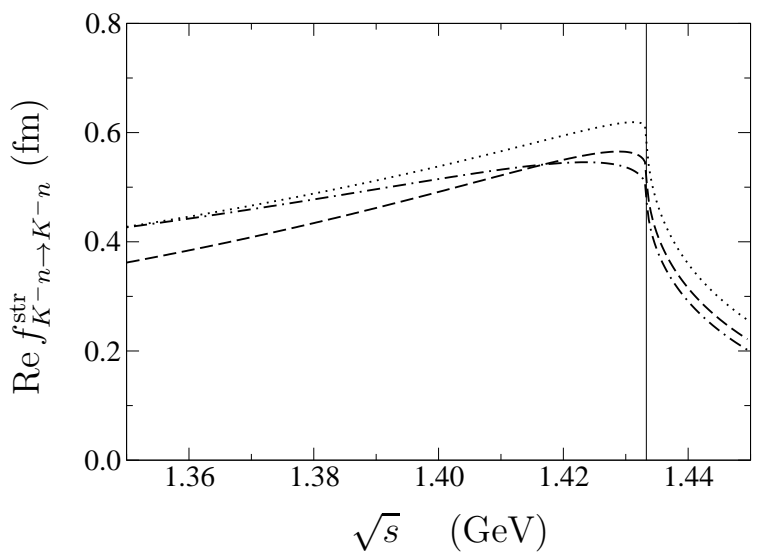

(a)

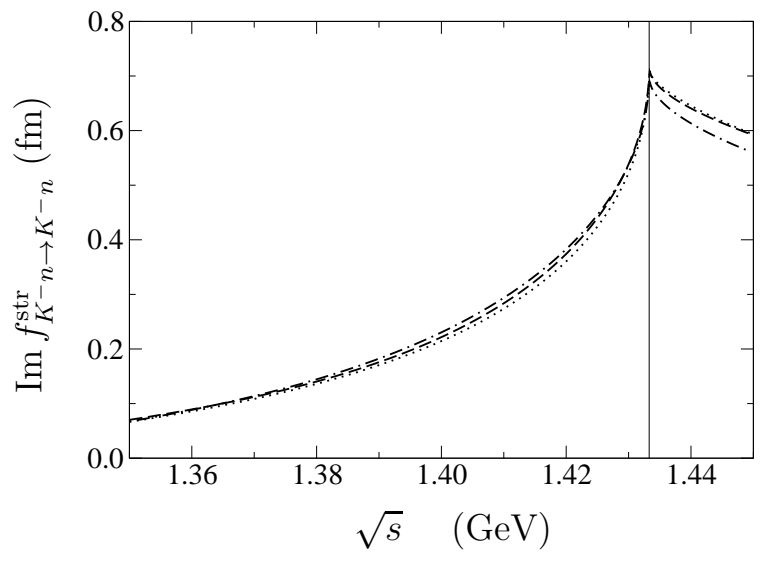

(b)

Figure 11: Real (left panel) and imaginary part (right panel) of the strong interaction ( $s$-wave) elastic $K^{-} n$ amplitude. The dashed, dotted and dot-dashed lines correspond to the approaches "WT", "c" and "s", respectively. The $K^{-} n$ threshold is indicated by the vertical line.

\begin{tabular}{|c|c|c|c|}
\hline & "WT" & "c" & "s" \\
\hline$a_{K^{-n}} \quad(\mathrm{fn}$ & $0.53+0.72 i$ & $0.61+0.71 i$ & $0.49+0.70$ \\
\hline
\end{tabular}

Table 7: $K^{-} n$ scattering lengths for the approaches "WT", "c" and " $s$ ".

\section{3 $\quad K^{-} n$ and $K^{+} p$ scattering}

Once the parameters have been fixed from $K^{-} p$ data, the same approach provides predictions for $K^{-} n$ scattering, since no new unknown constants appear. The real and imaginary parts of the elastic $s$-wave $K^{-} n$ scattering amplitude are presented in Fig. 11 for the "WT", "c" and "s" frameworks. All three versions yield similar results and the predicted scattering lengths given in Table 7 are consistent with the empirical value $a_{K^{-}{ }_{n}} \sim 0.4+i 0.6 \mathrm{fm}$ [38] within errors.

We have refrained from presenting results for the " $u$ "-approach for $K^{-} n \rightarrow K^{-} n$. The reason is the appearance of an unphysical subthreshold cut in the $\eta \Sigma^{-}$channel at $1.426 \mathrm{GeV}$ just below the $K^{-} n$ threshold. As already mentioned in Sec. 2.2, this is an artifact of the onshell formalism which would not be present in a full field theoretical calculation. The previously applied procedure of eliminating the unphysical subthreshold cut by matching the contribution of the crossed Born diagram to a constant value below a certain invariant energy $\sqrt{s_{0}}$ does not work here since the singularity at $1.426 \mathrm{GeV}$ is just $7 \mathrm{MeV}$ away from $K^{-} n$ threshold.

Finally, we note that in the $K^{+}$-proton channel, the different approaches ("WT", "c" and "3") yield scattering lengths in the range $a_{K^{+} p} \simeq-(0.26 \ldots 0.36) \mathrm{fm}$, consistent with the empirical $a_{K^{+} p} \simeq-0.33 \mathrm{fm}[38]$.

\subsection{Coulomb effects}

For small incident kaon momenta close to $\bar{K} N$ threshold, the elastic $K^{-} p$ scattering cross section receives sizable contributions from both the strong and the electromagnetic interaction. Coulomb interactions are taken into account by utilizing the quantum mechanical Coulomb scattering amplitude Eq. (14). Due to the infinite-range nature of the Coulomb potential, the scattering amplitude is infrared divergent in the limit of small incident momenta as well as 


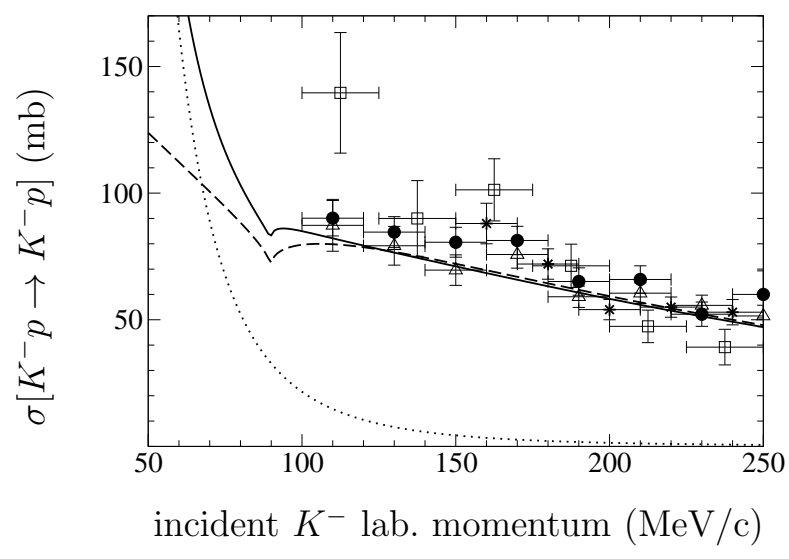

(a)

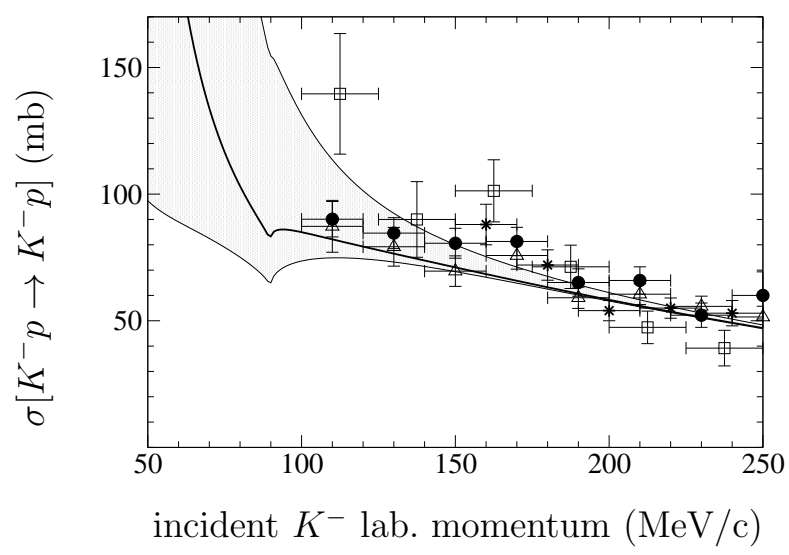

(b)

Figure 12: Left: Contributions to the total elastic cross sections of $K^{-} p$ scattering from Coulomb interaction (dotted), strong interaction (dashed) and their coherent sum (solid). Right: Dependence on the small-angle cutoff excluding small center-of-mass angles. The lower boundary of the band corresponds to $\cos \theta_{\min }=0.7$, the upper one to $\cos \theta_{\min }=0.99$; the solid line represents the value established by the experiments [23,24], which we also use in our calculations: $\cos \theta_{\min }=0.966$.

small scattering angles.

As explained in section 2.3, the divergence at $\mathbf{q}_{\mathrm{cm}}=0$ can be ignored in the energy regime accessible by the scattering experiments. However, when performing the integration over the center-of-mass scattering angle in order to calculate the total elastic cross section a cutoff in the angle must be introduced. Two of the experiments that have produced data at the lowest kaon momenta, exclude forward scattering angles and consider only the range $-1 \leq \cos \theta_{c m} \leq$ $0.966[23,24]$. We choose to work with the same angle cutoff in order to perform consistent comparisons. The contributions of the Coulomb and the strong interaction as well as their coherent sum are displayed in an exemplary case for fit "1" in Fig. 12a. While the corrections due to the Coulomb interaction are completely negligible for kaon laboratory momenta greater than $150 \mathrm{MeV} / c$, they start becoming important below $100 \mathrm{MeV} / c$.

In Fig. 12b we show the dependence of our results on the small-angle cut. The gray band indicates the variation between $\cos \theta_{\min }=0.7$, so that the Coulomb amplitude is highly suppressed, and $\cos \theta_{\min }=0.99$ where it is sizable. The curves have been normalized to the solid angle covered by the experiments [23,24]. For large incident kaon momenta (above $150 \mathrm{MeV} / \mathrm{c}$ ) where the strong $s$-wave amplitude dominates, the omission of forward scattering angles makes the elastic cross section decrease by only a few percent when compared with the integration over the full solid angle. It is therefore justified to compare our results directly with all experimental data, given their large error spread.

\subsection{Resonance poles}

Finally, we turn our attention to the poles of the strong interaction $T$ matrix in the complex $W \equiv \sqrt{s}$ plane. These poles are usually classified according to their isospin and we keep the notation of $I=0$ and $I=1$ poles even though we work in the physical basis where isospin is broken by the physical masses of the particles. Although we observe two poles in the unphysical 
sheet which is directly connected to the physical region between the $\pi \Sigma$ and $\bar{K} N$ thresholds, their positions depend strongly on the chosen approach. As a matter of fact, the formation of a pronounced double pole structure close to the real axis as reported in [8] occurs only in the "WT" model. When next-to-leading order corrections are taken into account the second pole is shifted further away from the real axis and its contribution to the physical region tends to dissolve in the background.

Following [8] we define complex parameters $g_{i}$ representing the contribution to the coupling strength at the pole from the channel with index $i^{4}$ They can be extracted by the residue of the $T$ matrix at the position $W_{0}$ of the pole

$$
g_{i} g_{j}=\operatorname{Res}_{W_{0}} T_{i j} .
$$

Since the $T$ matrix is merely defined up to an arbitrary complex phase, only the modulus of $g_{i}$ is meaningful. In Table 8 we show the positions and coupling strengths of the $\Lambda(1405)$ poles classified according to the different approaches and fits. For clarity, the pole positions are also depicted in Fig. 13. A result common to all approaches is the fact that the pole which couples strongly to the $I=0 \bar{K} N$ state (see open symbols in Fig. 13) is located closer to the real axis, in agreement with [8]. The inclusion of higher order contact terms slightly lowers its real part, whereas its position is practically not affected when the direct and crossed Born terms are taken into account (approaches " $s$ " and " $u$ "), cf. Fig. 13a. The second pole which couples strongly to the $\pi \Sigma$ channels is shifted drastically by going from the "WT" to the "c" approach. It moves up in energy (even above the $\bar{K} N$ thresholds) and away from the real axis. The inclusion of the $s$ - and $u$-channel Born terms successively brings the pole to lower energies again. In the " $u$ " approach it is almost in line with the first pole, but located at quite some distance from the real axis, not supporting a pronounced double pole structure close to the real axis.

In order to study the interplay of the two poles and their effect on the real axis and thus on physical observables, we construct a simple model as done in [8]. If the $T$ matrix were solely furnished by two $\Lambda(1405)$ poles, it would be given by

$$
T_{i j}^{(\text {poles })}=\frac{g_{i}^{(1)} g_{j}^{(1)}}{W-W_{0}^{(1)}}+\frac{g_{i}^{(2)} g_{j}^{(2)}}{W-W_{0}^{(2)}},
$$

where the superscripts (1), (2) refer to the pertinent poles. In Fig. 14 we compare this simple pole amplitude to the full coupled channel $T$ matrix in the relevant $I=0 \pi \Sigma \rightarrow \pi \Sigma$ and $\bar{K} N \rightarrow \pi \Sigma$ channels by plotting the quantity $\left|\mathbf{q}_{c m}^{\pi \Sigma}\right|\left|T_{\pi \Sigma, \bar{K} N \rightarrow \pi \Sigma}\right|^{2}$, where $T$ is given by either just one pole, both poles, or by the full coupled channel result. For illustrative purposes we restrict ourselves to the "WT" and "c" models. Both for the "WT" and "c" approach the $\bar{K} N \rightarrow \pi \Sigma$ amplitude is dominated by the pole close to the real axis, which couples most strongly to the $\bar{K} N$ states, and the full $T$ matrix element is well described by the pole model. For the process $\pi \Sigma \rightarrow \pi \Sigma$ the biggest portion of the "WT" result stems again from the pole contributions, reflecting the double pole structure close to the real axis. In contrast, the inclusion of $\mathcal{O}\left(p^{2}\right)$ contact terms in the "c" approach significantly reduces the influence of the second pole which couples mainly to the $I=0$ combination of $\pi \Sigma$ channels, giving rise to a large background contribution to the amplitude. This is also observed for the " $s$ " and " $u$ " approaches. Note also that due to interference effects the subthreshold peak of the $\pi \Sigma \rightarrow \pi \Sigma$ pole model amplitude

\footnotetext{
${ }^{4}$ Note that our definition of the $T$ matrix differs from that in [8] by a factor of $-2 \sqrt{M_{a} M_{b}}$.
} 


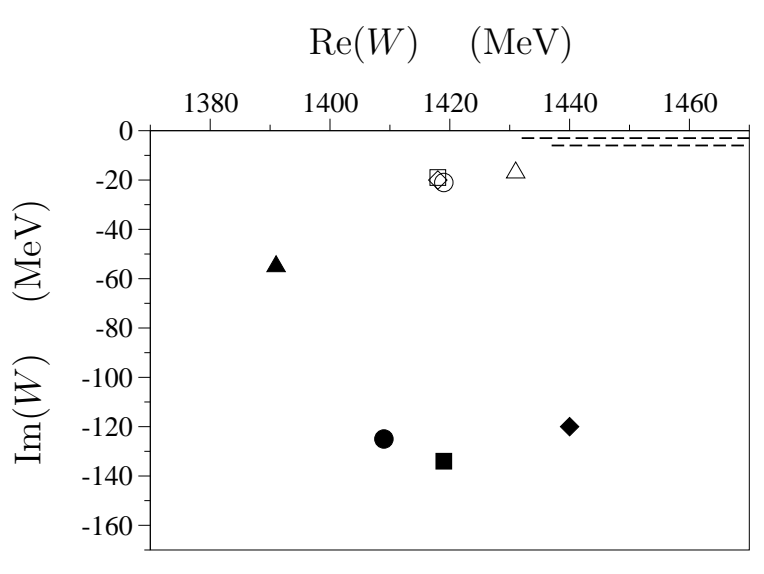

(a)

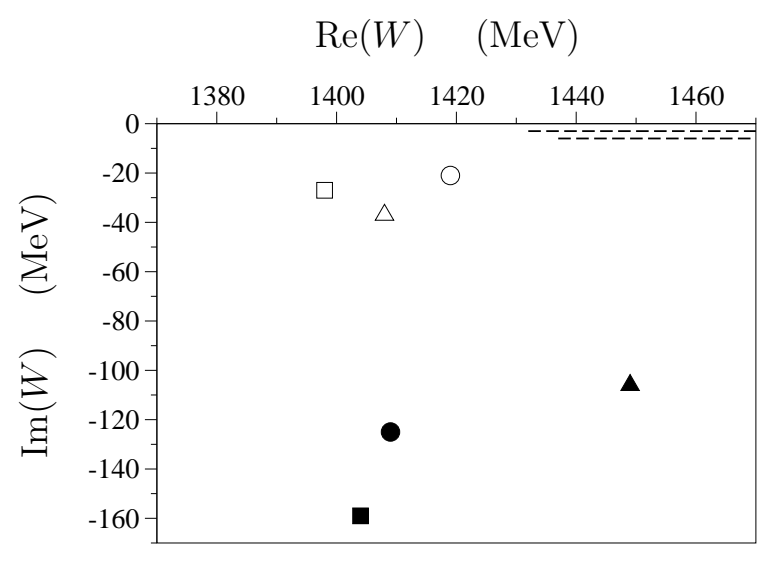

(b)

Figure 13: Left: Pole positions of the $T$ matrix in the complex $W$ plane. The triangles, diamonds, squares and circles correspond to the "WT", "c", "s" and " $u$ " approach, respectively. The dashed lines represent the $K^{-} p$ and $\bar{K}^{0} n$ cuts, respectively. Right: Pole positions of the $T$ matrix in the complex $W$ plane. The circles, triangles and squares correspond to the fits "1", "2" and "3", respectively.

appears at a similar position in both the "WT" and the "c" approach, although the second pole happens to be located at quite different positions in the complex plane.

For completeness, we also show in Table 8 and Fig. 13 the pole positions and couplings extracted from the fits "2" and "3", obtained by approaching the constraints set by the DEAR experiment. Again the pole with strong coupling to $\bar{K} N$ is located close to the real axis, and its real part is decreased by going from fit "1" to fit " 3 ". The position of the second pole varies a lot with a strong tendency to further depart from the real axis. Furthermore, we note that the characteristic strong coupling of the second pole to $\pi \Sigma$ states is equaled in magnitude by the coupling to the $\bar{K} N$ channel when the DEAR data are taken into account. The key feature, independent of the additional constraint imposed by the DEAR data, is that the contribution from the second pole on the real axis dissolves in the background once next-to-leading order $\left(\mathcal{O}\left(p^{2}\right)\right)$ dynamics are turned on in addition to the leading Weinberg-Tomozawa term.

We conclude that although the different approaches yield similar fits to all available experimental data, the pertinent pole structures are quite diverse. For the "WT" version we observe a pronounced double pole structure as described in [8]. For the schemes which include higher order contact terms, only the pole which couples most strongly to the $\bar{K} N$ state is located close to the real axis. The influence of the second pole is substantially reduced and the $T$ matrix cannot be well approximated by the outlined pole model. Instead, background contributions are important. Our findings emphasize that the analytic continuation of partial waves in the complex energy plane depends sensitively on the basic dynamical input of the underlying chiral $\mathrm{SU}(3)$ Lagrangian.

\section{Conclusions}

In the present work, we have critically examined and updated the analysis of the $\bar{K} N$ system within the framework of coupled-channels approaches combined with chiral SU(3) dynamics. There is renewed interest in the investigation of the $\bar{K} N$ channel in the light of the new accurate measurement of the strong interaction shift and width of kaonic hydrogen at DEAR which sets 

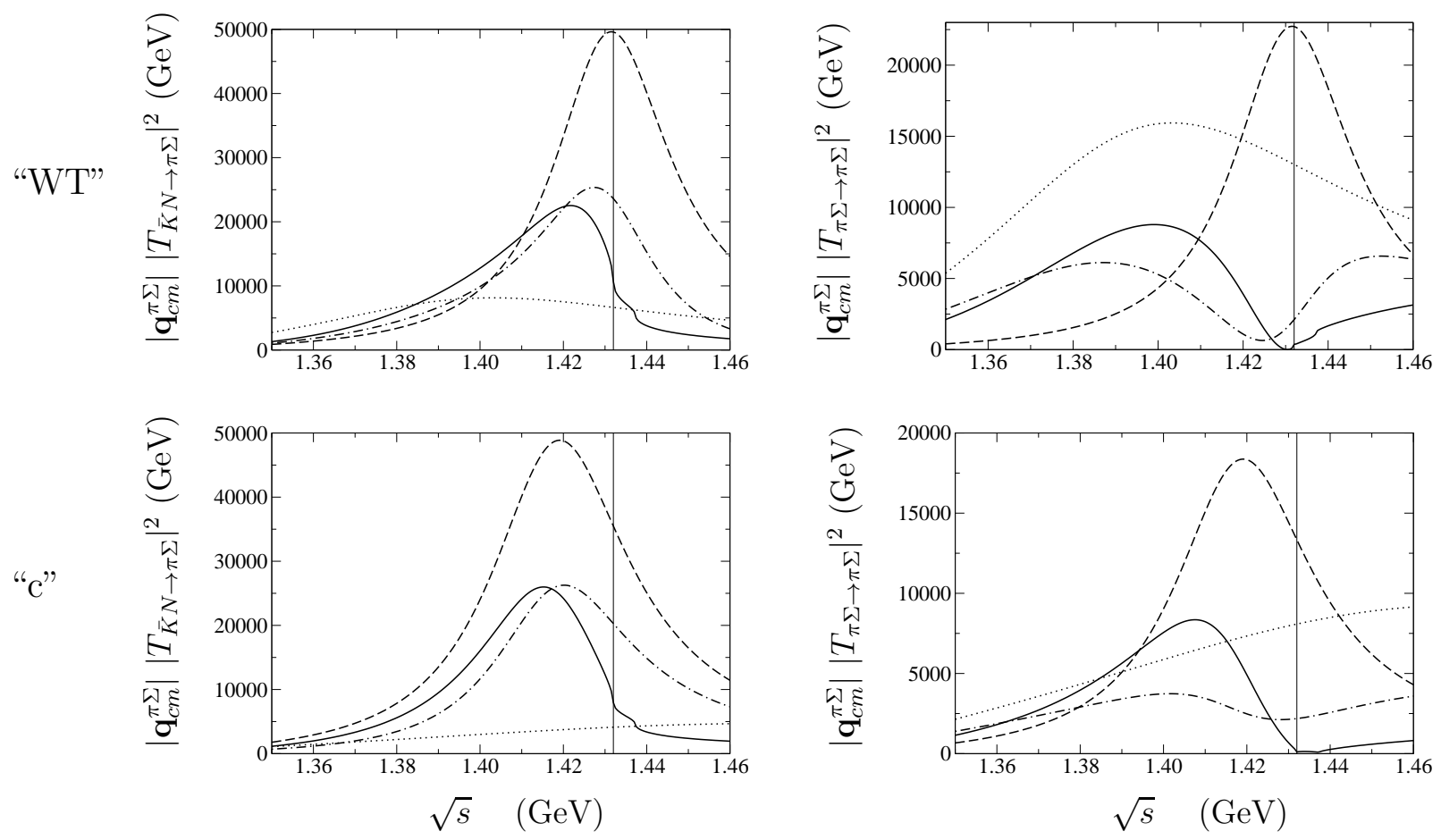

Figure 14: Comparison of the pole model described in the text and the full coupled channel $T$ matrix for the approaches "WT" (upper figures) and "c" (lower figures). We plot the quantities $\left|\mathbf{q}_{c m}^{\pi \Sigma}\right|\left|T_{\bar{K} N \rightarrow \pi \Sigma}\right|^{2}$ (left column) and $\left|\mathbf{q}_{c m}^{\pi \Sigma}\right|\left|T_{\pi \Sigma \rightarrow \pi \Sigma}\right|^{2}$ (right column) for $I=0$ meson-baryon states. The lines represent the contribution of the first pole (dashed), the second pole (dotted), both (dot-dashed), and the full coupled-channels result (solid).

\begin{tabular}{|c|l|c|c|c|c|}
\hline & & \multicolumn{4}{|c|}{$\left|g_{i}\right|$} \\
& $W_{0}(\mathrm{MeV})$ & $\pi \Sigma$ & $\bar{K} N$ & $\eta \Lambda$ & $K \Xi$ \\
\hline "WT" & $1431-17 i$ & 2.46 & 3.64 & 1.82 & 0.49 \\
& $1391-55 i$ & 4.29 & 3.06 & 0.84 & 0.81 \\
\hline "c" & $1418-20 i$ & 2.59 & 4.22 & 1.98 & 0.72 \\
& $1440-120 i$ & 5.05 & 3.60 & 1.69 & 1.63 \\
\hline "s" & $1418-19 i$ & 2.46 & 4.29 & 2.13 & 0.60 \\
& $1419-134 i$ & 5.02 & 3.72 & 1.49 & 1.73 \\
\hline "u" "1" & $1419-21 i$ & 2.68 & 4.42 & 2.27 & 0.36 \\
& $1409-125 i$ & 5.13 & 3.99 & 1.90 & 1.10 \\
\hline "2" & $1408-37 i$ & 4.19 & 5.55 & 3.28 & 0.49 \\
& $1449-106 i$ & 6.16 & 6.12 & 4.25 & 1.39 \\
\hline "3" & $1398-27 i$ & 3.08 & 4.86 & 2.58 & 1.55 \\
& $1404-159 i$ & 4.58 & 4.57 & 2.97 & 1.17 \\
\hline
\end{tabular}

Table 8: Positions of the poles which are relevant for $\Lambda(1405)$ and their coupling strengths to isospin $I=0$ states. 
tight constraints. It is therefore worth investigating whether both the DEAR data and the $K^{-} p$ scattering data can be accommodated by coupled-channels analyses, while at the same time trying to reduce the inherent model dependence of these approaches wherein chiral effective field theory is combined with a non-perturbative Bethe-Salpeter equation. The driving terms for the Bethe-Salpeter equation are derived from the effective Lagrangian and constitute a major source of model dependence. Several variants of such approaches are commonly used in the literature, e.g., only the Weinberg-Tomozawa term originating from the leading order Lagrangian is taken into account, while in other works direct and crossed Born terms are added or contact interactions of the next-to-leading chiral order are included.

In the present investigation, we have worked out the driving terms in four consecutive steps. Starting from the Weinberg-Tomozawa term, we successively added contact interactions of second chiral order, the direct Born term and the crossed Born term.

All four versions have in common that the agreement with $\bar{K} N$ scattering data is partly spoilt once the new tight constraints imposed by the DEAR experiment are taken into account. (We mention though that the results of these models fall within the larger error ranges of the KEK experiment.) The largest discrepancies are observed in the elastic $K^{-} p$ channel where the calculated cross section is substantially lowered by inclusion of the DEAR data. Coulomb effects ameliorate the situation at low kaon laboratory momenta below $100 \mathrm{MeV} / c$, but an offset to elastic $K^{-} p$ scattering data remains. Moreover, electromagnetic corrections to the strong interaction shift and width in kaonic hydrogen as given in [35] reduce the discrepancy further, but are not able to compensate the difference between the coupled-channels approaches and the experimental data. Further tight phenomenological constraints in the $\bar{K} N$ system are provided by the threshold branching ratios which have been measured very precisely. Inclusion of the DEAR data produces results for the branching ratio $R_{c}$ which are not in agreement with the quoted experimental error ranges.

Another consistency check could be provided by studying the $K^{-} p \rightarrow \pi \Sigma$ amplitude below the $K^{-} p$ threshold, since inclusion of the DEAR data amounts to a substantial change in this amplitude. Experiments towards this direction are currently analyzed at SPring-8/LEPS and at ELSA, where photoproduction of $\Lambda(1405)$ has been measured. If $K^{-}$exchange in the $t$ channel can be isolated from these data, the information gained would be very useful in order to set constraints for the $\bar{K} N$ scattering amplitude below threshold [37].

The comparison between the different variants of the coupled-channels approaches can be summarized as follows. The quality of the fits to data is improved substantially by including next-to-leading order contact interactions with new parameters of the effective Lagrangian which we vary within reasonable ranges as explained in the text. The inclusion of the Born terms, on the other hand, leads only to minor changes. The treatment of the interaction kernel at subleading order also destroys the pronounced double pole structure of the $\Lambda(1405)$ close to the real axis as observed in [8]. Although we still see two poles in the relevant unphysical sheet, the pole with a stronger coupling to the $\pi \Sigma$ channel now moves far away from the real axis, losing its importance for any physical observables. As a consequence, the full partial wave amplitude for $\pi \Sigma \rightarrow \pi \Sigma$ is not approximated well by just these two poles and the background contribution becomes important.

The updated, constrained analysis presented here is also of considerable interest in the discussion of possible deeply bound $K^{-}$- nuclear states [39]. The amplitudes shown in Figs. 9, 10 suggest a complex, energy dependent subthreshold $\bar{K}$-nucleus potential which is attractive in the $\bar{K} N$ energy range below the $\Lambda(1405)$. Its imaginary part decreases as the energy is lowered 
towards the $\pi \Sigma$ threshold, an effect that has been pointed out previously in Refs. [40]. This is a potential mechanism for supporting narrow bound $\bar{K}$ states at sufficiently large nuclear densities, but details concerning the strong energy dependence of the driving potentials require additional constraints and further investigation.

\section{Acknowledgments}

We thank M. Lutz and U.-G. Meißner for useful discussions. Partial financial support by DFG and BMBF is gratefully acknowledged. This research is part of the EU Integrated Infrastructure Initiative Hadronphysics under contract number RII3-CT-2004-506078.

\section{A Tree level amplitudes}

The amplitudes for the meson-baryon scattering processes $\phi_{i} B_{a}^{\sigma} \rightarrow \phi_{j} B_{b}^{\sigma^{\prime}}$ (with spin indices $\sigma$, $\left.\sigma^{\prime}\right)$ corresponding to the tree level diagrams in Fig. 1a, c, d have already been given in [5], but for completeness we present them here in our notation along with the next-to-leading order contact term depicted in Fig. 1b. One obtains

$$
\begin{aligned}
& V_{j b, i a}^{(a)}=\frac{1}{8 f^{2}} C_{j b, i a}^{(a)} N_{a} N_{b} \\
& \times\left(\chi_{b}^{\sigma^{\prime}}\right)^{T}\left[2 \sqrt{s}-M_{a}-M_{b}+\left(2 \sqrt{s}+M_{a}+M_{b}\right) \frac{\mathbf{q}^{\prime} \cdot \mathbf{q}+i\left(\mathbf{q}^{\prime} \times \mathbf{q}\right) \cdot \boldsymbol{\sigma}}{N_{a}^{2} N_{b}^{2}}\right] \chi_{a}^{\sigma}, \\
& V_{j b, i a}^{(b)}=\frac{-1}{f^{2}}\left(C_{j b, i a}^{\left(b_{1}\right)}-2\left(E_{i} E_{j}-\mathbf{q}^{\prime} \cdot \mathbf{q}\right) C_{j b, i a}^{\left(b_{2}\right)}\right) N_{a} N_{b}\left(\chi_{b}^{\sigma^{\prime}}\right)^{T}\left[1-\frac{\mathbf{q}^{\prime} \cdot \mathbf{q}+i\left(\mathbf{q}^{\prime} \times \mathbf{q}\right) \cdot \boldsymbol{\sigma}}{N_{a}^{2} N_{b}^{2}}\right] \chi_{a}^{\sigma} \\
& V_{j b, i a}^{(c)}=\frac{-1}{12 f^{2}} \sum_{c=1}^{8} C_{j b, c}^{(c)} C_{i a, c}^{(c)} N_{a} N_{b} \frac{1}{s-M_{c}^{2}} \\
& \times\left(\chi_{b}^{\sigma^{\prime}}\right)^{T}\left[\left(\sqrt{s}-M_{a}\right)\left(s-\left(M_{b}+M_{c}\right) \sqrt{s}+M_{b} M_{c}\right)\right. \\
& \left.+\left(\sqrt{s}+M_{a}\right)\left(s+\left(M_{b}+M_{c}\right) \sqrt{s}+M_{b} M_{c}\right) \frac{\mathbf{q}^{\prime} \cdot \mathbf{q}+i\left(\mathbf{q}^{\prime} \times \mathbf{q}\right) \cdot \boldsymbol{\sigma}}{N_{a}^{2} N_{b}^{2}}\right] \chi_{a}^{\sigma}, \\
& V_{j b, i a}^{(d)}=\frac{1}{12 f^{2}} \sum_{c=1}^{8} C_{i c, b}^{(c)} C_{j c, a}^{(c)} N_{a} N_{b} \frac{1}{u-M_{c}^{2}}\left(\chi_{b}^{\sigma^{\prime}}\right)^{T} \\
& \times\left[u\left(\sqrt{s}+M_{c}^{2}\right)+\sqrt{s}\left(M_{b}\left(M_{a}+M_{c}\right)+M_{a} M_{c}\right)-M_{b}\left(M_{a}+M_{c}\right)\left(M_{a}+M_{b}\right)-M_{a}^{2} M_{c}\right. \\
& +\left(u\left(\sqrt{s}-M_{c}^{2}\right)+\sqrt{s}\left(M_{b}\left(M_{a}+M_{c}\right)+M_{a} M_{c}\right)+M_{b}\left(M_{a}+M_{c}\right)\left(M_{a}+M_{b}\right)+M_{a}^{2} M_{c}\right) \\
& \left.\times \frac{\mathbf{q}^{\prime} \cdot \mathbf{q}+i\left(\mathbf{q}^{\prime} \times \mathbf{q}\right) \cdot \boldsymbol{\sigma}}{N_{a}^{2} N_{b}^{2}}\right] \chi_{a}^{\sigma} .
\end{aligned}
$$


The two-component Pauli-spinor of a baryon $B$ with spin $\sigma$ is symbolized by $\chi_{B}^{\sigma}$ while the pertinent normalization factor is given by $N_{B}=\sqrt{E_{B}+M_{B}}$ and $E_{x}$ is the center-of-mass energy of particle $x$. The center-of-mass three-momenta of the initial and final particles are denoted by $\mathbf{q}$ and $\mathbf{q}^{\prime}$, respectively. The Mandelstam variable $u$ is given by $u=\left(p-k^{\prime}\right)^{2}$, where $p$ is the four-momentum of the initial baryon and $k^{\prime}$ that of the final meson. The coefficients $C_{j b, i a}^{(a)}, C_{j b, i a}^{\left(b_{1}\right)}$ and $C_{j b, i a}^{\left(b_{2}\right)}$, which are symmetric under the interchange of initial and final mesonbaryon pairs, are compiled in Tables 9, 10 and 11, respectively, whereas the non-zero axial vector couplings $C_{\phi B_{1}, B_{2}}^{(c)}$ (which are symmetric under the combined transformation $B_{1} \leftrightarrow B_{2}$ and $\phi \leftrightarrow \bar{\phi})$ are given by

$$
\begin{aligned}
& C_{K^{-}, \Lambda}^{(c)}=C_{\bar{K}^{0} n, \Lambda}^{(c)}=C_{\eta \Xi^{-}, \Xi^{-}}^{(c)}=C_{\eta \Xi^{0}, \Xi^{0}}^{(c)}=-D-3 F, \\
& \sqrt{2} C_{K^{-} p, \Sigma^{0}}^{(c)}=-\sqrt{2} C_{\bar{K}^{0} n, \Sigma^{0}}^{(c)}=C_{\bar{K}^{0} p, \Sigma^{+}}^{(c)}=C_{K^{-} n, \Sigma^{-}}^{(c)}=C_{\pi^{+} \Xi^{-}, \Xi^{0}}^{(c)}=\sqrt{2} C_{\pi^{0} \Xi^{-}, \Xi^{-}} \\
& \quad=-\sqrt{2} C_{\pi^{0} \Xi^{0}, \Xi^{0}}=\sqrt{6}(D-F), \\
& C_{\pi^{0} \Sigma^{0}, \Lambda}^{(c)}=C_{\pi^{+} \Sigma^{-}, \Lambda}^{(c)}=C_{\pi^{-} \Sigma^{+}, \Lambda}^{(c)}=C_{\eta \Sigma^{+}, \Sigma^{+}}^{(c)}=C_{\eta \Sigma^{-}, \Sigma^{-}}^{(c)}=C_{\eta \Sigma^{0}, \Sigma^{0}}^{(c)}=-C_{\eta \Lambda, \Lambda}^{(c)}=2 D, \\
& C_{\pi^{+} \Sigma^{-}, \Sigma^{0}}^{(c)}=-C_{\pi^{-} \Sigma^{+}, \Sigma^{0}}^{(c)}=-C_{\pi^{0} \Sigma^{-}, \Sigma^{-}}^{(c)}=C_{\pi^{0} \Sigma^{+}, \Sigma^{+}}^{(c)}=2 \sqrt{3} F, \\
& C_{K^{+} \Xi^{-}, \Lambda}^{(c)}=C_{K^{0} \Xi^{0}, \Lambda}^{(c)}=C_{\eta p, p}^{(c)}=C_{\eta n, n}^{(c)}=-D+3 F, \\
& \sqrt{2} C_{K^{+} \Xi^{-}, \Sigma^{0}}^{(c)}=-\sqrt{2} C_{K^{0} \Xi^{0}, \Sigma^{0}}^{(c)}=C_{\pi^{-} p, n}^{(c)}=\sqrt{2} C_{\pi^{0} p, p}^{(c)}=-\sqrt{2} C_{\pi^{0} n, n}^{(c)}=C_{\bar{K}^{0} \Sigma^{-}, \Xi^{-}}^{(c)} \\
& \quad=C_{K^{-} \Sigma^{+}, \Xi^{0}}^{(c)}=\sqrt{6}(D+F) .
\end{aligned}
$$

The interaction kernels utilized in the various approaches under consideration, "WT", "c", "s" and " $u$ ", are obtained by projecting out the $s$-wave part of the amplitudes according to Eq. (8) with

$$
\begin{aligned}
V_{\text {"WT" }} & =V^{(a)} \\
V_{\text {"c" }} & =V^{(a)}+V^{(b)} \\
V_{\text {"s" }} & =V^{(a)}+V^{(b)}+V^{(c)} \\
V_{\text {"u }} & =V^{(a)}+V^{(b)}+V^{(c)}+V^{(d)} .
\end{aligned}
$$

\section{References}

[1] N. Kaiser, P. B. Siegel, and W. Weise, Nucl. Phys. A594 (1995) 325.

[2] E. Oset, A. Ramos, Nucl. Phys. A635 (1998) 99.

[3] N. Kaiser, P. B. Siegel, and W. Weise, Phys. Lett. B362 (1995) 23;

N. Kaiser, T. Waas, and W. Weise, Nucl. Phys. A612 (1997) 297;

J. Caro Ramon, N. Kaiser, S. Wetzel, and W. Weise, Nucl. Phys. A672 (2000) 249. 


\begin{tabular}{|c|c|c|c|c|c|c|c|c|c|c|}
\hline & $K^{-} p$ & $\bar{K}^{0} n$ & $\pi^{0} \Lambda$ & $\pi^{0} \Sigma^{0}$ & $\pi^{+} \Sigma^{-}$ & $\pi^{-} \Sigma^{+}$ & $\eta \Lambda$ & $\eta \Sigma^{0}$ & $K^{-} \Xi^{+}$ & $K^{0} \Xi^{0}$ \\
\hline$K^{-} p$ & 4 & 2 & $\sqrt{3}$ & 1 & 0 & 2 & 3 & $\sqrt{3}$ & 0 & 0 \\
\hline $\bar{K}^{0} n$ & & 4 & $-\sqrt{3}$ & 1 & 2 & 0 & 3 & $-\sqrt{3}$ & 0 & 0 \\
\hline$\pi^{0} \Lambda$ & & & 0 & 0 & 0 & 0 & 0 & 0 & $\sqrt{3}$ & $-\sqrt{3}$ \\
\hline$\pi^{0} \Sigma^{0}$ & & & & 0 & 4 & 4 & 0 & 0 & 1 & 1 \\
\hline$\pi^{+} \Sigma^{-}$ & & & & & 4 & 0 & 0 & 0 & 2 & 0 \\
\hline$\pi^{-} \Sigma^{+}$ & & & & & & 4 & 0 & 0 & 0 & 2 \\
\hline$\eta \Lambda$ & & & & & & & 0 & 0 & 3 & 3 \\
\hline$\eta \Sigma^{0}$ & & & & & & & & 0 & $\sqrt{3}$ & $-\sqrt{3}$ \\
\hline$K^{-} \Xi^{+}$ & & & & & & & & & 4 & 2 \\
\hline$K^{0} \Xi^{0}$ & & & & & & & & & & 4 \\
\hline
\end{tabular}

Table 9: Coefficients $C_{j b, i a}^{(a)}=C_{i a, j b}^{(a)}$ of the leading-order contact interaction.

[4] B. Krippa, Phys. Rev. C58 (1998) 1333.

[5] J. A. Oller and U.-G. Meißner, Phys. Lett. B500 (2001) 263.

[6] M. F. M. Lutz and E. Kolomeitsev, Nucl. Phys. A700 (2002) 193.

[7] B. Borasoy, E. Marco, and S. Wetzel, Phys. Rev. C66 (2002) 055208.

[8] D. Jido, J. A. Oller, E. Oset, A. Ramos, and U. G. Meißner, Nucl. Phys. A725 (2003) 181.

[9] A. Bahaoui, C. Fayard, T. Mizutani, and B. Saghai, Phys. Rev. C68 (2003) 064001.

[10] M. Cargnelli et al. (DEAR collaboration), Int. J. Mod. Phys. A20 (2005) 341.

[11] M. Iwasaki et al., Phys. Rev. Lett. 78 (1997) 3067;

T. M. Ito et al., Phys. Rev. C58 (1998) 2366.

[12] B. Borasoy, R. Nißler, and W. Weise, hep-ph/0410305.

[13] C. Garcia-Recio, M. F. M. Lutz, and J. Nieves, Phys. Lett. B582 (2004) 49.

[14] C. Garcia-Recio, J. Nieves, E. Ruiz Arriola, and M. J. Vicente Vacas, Phys. Rev. D67 (2003) 076009.

[15] J. K. Ahn, Nucl. Phys. A721 (2003) 715c.

[16] J. Gasser and H. Leutwyler, Nucl. Phys. B250 (1985) 465.

[17] A. Krause, Helv. Phys. Acta 63 (1990) 3. 


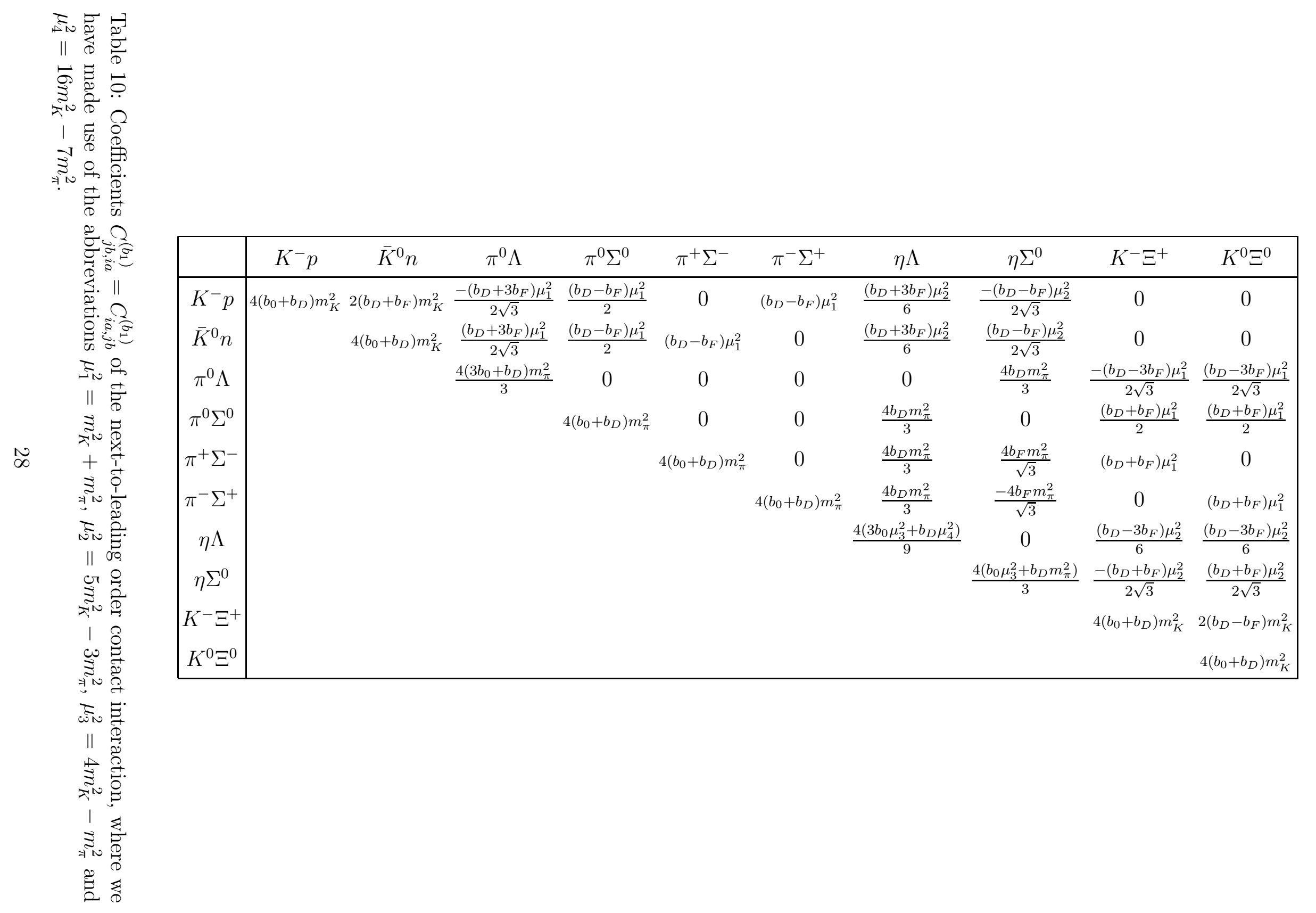




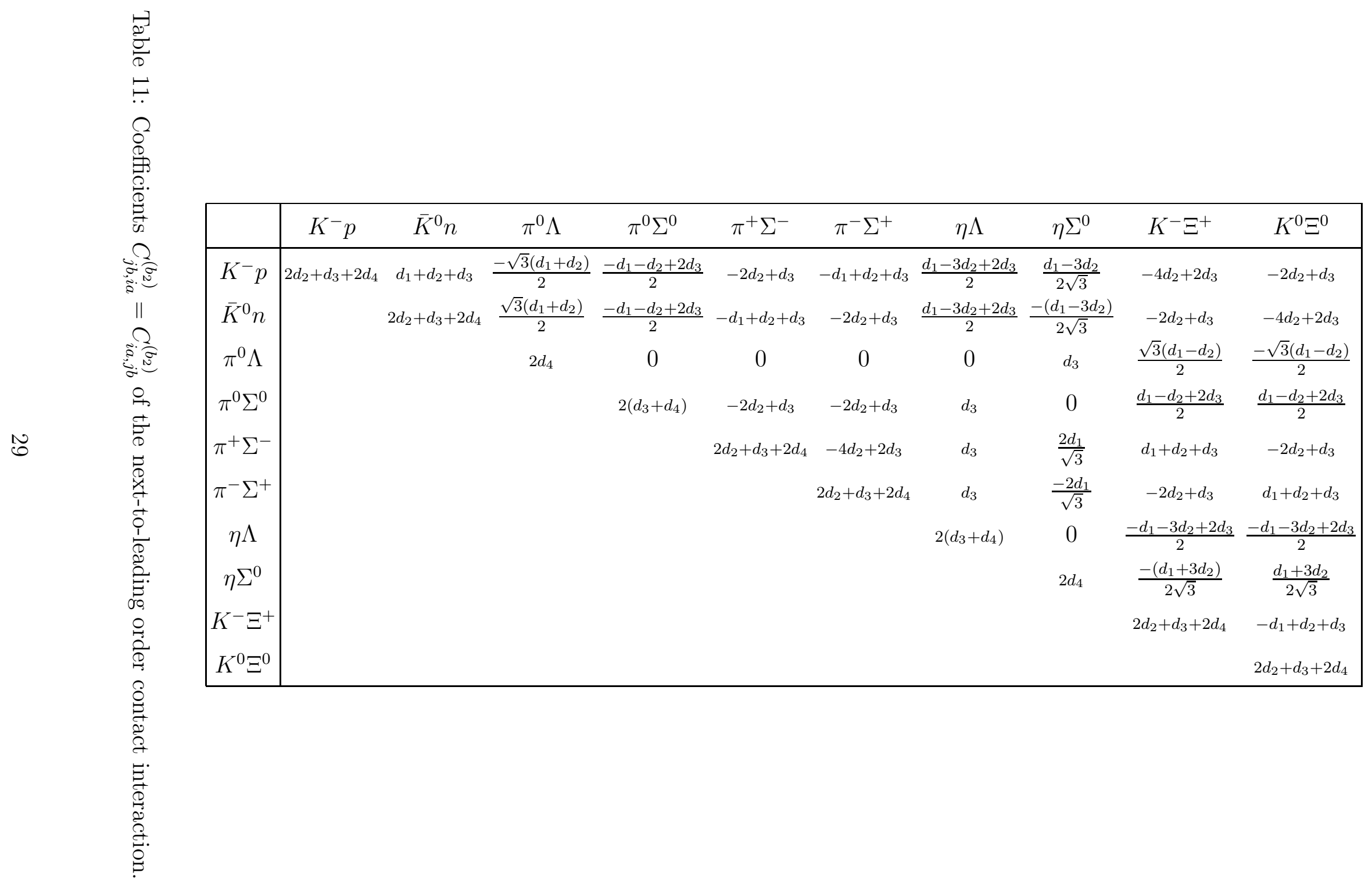


[18] F. E. Close and R. G. Roberts, Phys. Lett. B316 (1993) 165;

B. Borasoy, Phys. Rev. D 59 (1999) 054021.

[19] U.-G. Meißner and J. A. Oller, Nucl. Phys. A673 (2000) 311.

[20] G. Höhler, in: Landolt-Börnstein, vol. 9b2, ed. H. Schopper (Springer, Berlin, 1983) Appendix A7.

[21] J. C. Jackson and H. W. Wyld, Phys. Rev. Lett. 8 (1959) 355;

R. H. Dalitz and S. F. Tuan, Ann. Phys. 10 (1960) 307.

[22] L. D. Landau and E. M. Lifshitz, "Quantum Mechanics", Pergamon Press, Oxford (1977).

[23] W. E. Humphrey and R. R. Ross, Phys. Rev. 127 (1962) 1305.

[24] M. Sakitt et al., Phys. Rev. 139 (1965) B719.

[25] J. K. Kim, Phys. Rev. Lett. 14 (1965) 29; Columbia University Report, Nevis 149 (1966); Phys. Rev. Lett. 19 (1967) 1074.

[26] W. Kittel, G. Otter, and I. Wacek, Phys. Lett. 21 (1966) 349.

[27] D. Evans et al., J. Phys. G9 (1983) 885.

[28] J. Ciborowski et al., J. Phys. G8 (1982) 13.

[29] R. J. Nowak et al., Nucl. Phys. B139 (1978) 61.

[30] D. N. Tovee et al., Nucl. Phys. B33 (1971) 493.

[31] R. J. Hemingway, Nucl. Phys. B253 (1985) 742.

[32] J. Gasser and H. Leutwyler, Ann. Phys. 158 (1984) 142.

[33] B. Borasoy, Eur. Phys. J. C8 (1999) 121.

[34] R. H. Dalitz and A. Deloff, J. Phys. G17 (1991) 289.

[35] U.-G. Meißner, U. Raha, and A. Rusetsky, Eur. Phys. J. C35 (2004) 349.

[36] S. Deser, M. L. Goldberger, K. Baumann, and W. Thirring, Phys. Rev. 96 (1954) 774;

T. L. Trueman, Nucl. Phys. 26 (1961) 57.

[37] M. F. M. Lutz and M. Soyeur, Nucl. Phys. A748 (2005) 499.

[38] A. D. Martin, Nucl. Phys. B179 (1981) 33.

[39] A. Dote, Y. Akaishi, and T. Yamazaki, Prog. Theor. Phys. Suppl. 156 (2004) 184.

[40] T. Waas, N. Kaiser, and W. Weise, Phys. Lett. B379 (1996) 34;

T. Waas, M. Rho, and W. Weise, Nucl. Phys. A617 (1997) 449;

T. Waas and W. Weise, Nucl. Phys. A625 (1997) 287. 